\section{MÉTODO DE EVALUACIÓN SINTETIZADA PARA RIESGO \\ DE DESASTRES CON ENFOQUE DE ORDENAMIENTO TERRITORIAL (MESR): UNA APLICACIÓN PARA LA CIUDAD DE CALI, COLOMBIA}

María Eugenia Sevillano Rodríguez ${ }^{1^{*}}$

\section{RESUMEN}

El objetivo de este artículo es evaluar el riesgo por inundación en la ciudad de Santiago de Cali, Colombia. Durante el procedimiento se aplicó el Método de Evaluación Sintetizada para Riesgo de Desastres con Enfoque de Ordenamiento Territorial (MESR). A partir de este modelo, se integró desde el enfoque de construcción social factores de amenaza y vulnerabilidad sintetizada para valorar el riesgo y determinar la población e infraestructura que podría afectarse. Los resultados indican que el riesgo clasificado como alto ocupa el $6 \%$, el medio $38 \%$ y el bajo el $56 \%$. A causa de esto, 34 barrios, 37.202 personas y 28 asentamientos informales podrían ser afectados en la primera fase de la ocurrencia. La cartografía de riesgo de inundación coincide con las zonas que históricamente han sido afectadas y donde se ubica la población más vulnerable. La importancia de estos hallazgos reside en la utilidad que muestra la gestión del riesgo de desastre para guiar procesos más resilientes y sustentables en relación al ordenamiento territorial.

\section{PALABRAS CLAVES}

Amenazas, Vulnerabilidad, Riesgo, Inundaciones, Colombia

\section{SYNTHESIZED ASSESSMENT METHOD FOR DISASTER RISK WITH A LAND USE APPROACH (MESR) AN APPLICATION FOR THE CITY OF CALI, COLOMBIA}

\begin{abstract}
The objective of this article is to assess the risk of flooding in the city of Santiago de Cali, Colombia. During the study, the Synthetic Assessment Method for Disaster Risk with a Land Use Approach (MESR) was applied. Based on this model, synthesized hazard and vulnerability factors were integrated from the social construction approach to assess risk. This allows to determine the population and infrastructure that could be affected. Results indicate that the risk classified as 'high' is about 6 percent, 'medium' 38 percent, and 'low' 56 percent. This means that 34 neighborhoods, 37,202 people and 28 informal settlements could be affected during the initial phases of flood occurrence. The produced flood-risk map coincides with the areas that have historically been affected by floods, and where the most vulnerable residents tend to locate. The relevance of these findings lies in the usefulness for disaster risk management to guide resilient and more sustainable territorial planning processes.
\end{abstract}

\section{KEYWORDS}

Hazards, Vulnerability, Risk, Flood, Colombia

\begin{abstract}
1. Doctorado en Estudios Urbanos, Universidad Autónoma de Ciudad Juárez, Ciudad Juárez, México.
\end{abstract}

*Autor de correspondencia: mariasevillano14@hotmail. com

Identificador: http://revistareder.com/ $\underline{\text { handle-0719-8477-2020-070 }}$

\section{RECIBIDO}

12 de junio de 2020

\section{ACEPTADO}

12 de agosto de 2020

\section{PUBLICADO}

1 de enero de 2021

Formato cita Recomendada (APA): Sevillano Rodríguez, M.E. (2021). Método de Evaluación Sintetizada para Riesgo de Desastres con Enfoque de Ordenamiento Territorial (MESR):

Una Aplicación para la Ciudad de Cali, Colombia. Revista de Estudios

Latinoamericanos sobre Reducción del Riesgo de Desastres REDER, 5(1), 4669. http://revistareder.com/ handle-0719-8477-2020-070

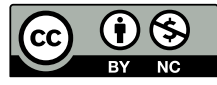

Todos los artículos publicados en REDER siguen una política de Acceso Abierto y se respaldan en una Licencia CreativeCommons Atribución-NoComercial 4.0 Internacional.

Revista de Estudios

Latinoamericanos sobre Reducción del Riesgo de Desastres (REDER)

Diseño: Lupe Bezzina 


\section{INTRODUCCIÓN}

En América Latina, las realidades principalmente urbanas relativas a la manifestación de los desastres han afectado a las poblaciones más pobres y vulnerables. Es importante precisar que todas las pérdidas humanas y materiales han propiciado estudios de riesgos y la necesidad de establecer procesos de gestión que reduzcan los daños (Ferradas-Mannucci \& Torres-Guevara, 2012). Si bien, el cambio climático ha sido un factor que contribuye a las inundaciones, debemos reconocer que estas manifestaciones no han sido extraordinarias de lo cual se cuenta con evidencia histórica (Linayo, 2012; Fernández \& Rodríguez, 1996). Esta consideración sustenta que la ocupación del territorio es una condición igual de importante que la modificación del clima (Linayo, 2012; Lampis \& Rubiano, 2012). Por consiguiente, existe una responsabilidad institucional, debido a que tienen la competencia de planificar y ordenar el desarrollo de entidades administrativas (Linayo, 2012). En relación con esto, la gestión de riesgo por inundación debe considerar aspectos relacionados con el peligro natural (lluvias frecuentes, regímenes de pluviosidad, modificación de cauces, etc.) y las condiciones de vulnerabilidad que enfrentan las poblaciones en relación a la fragilidad que los obliga a establecer asentamientos en zonas expuestas (riveras de ríos, lagunas, canales, etc.), así como a la capacidad de adaptarse a un clima cambiante (Lampis, 2013). De estas consideraciones, el interés mundial hacia temas de riesgos ha superado los estudios primordialmente biofísicos hacia la complejidad de variables de distinta procedencia -biofísicas y sociales- (Calderón-Ramírez \& Frey, 2017).

América Latina ha resultado ser un escenario propicio en vista de que concentra una importante población urbana, una gran variedad de amenazas de origen natural, vulnerabilidades, crecimiento urbano descontrolado y asentamientos informales (Watanabe, 2015; Sandoval \& Sarmiento, 2018). Conviene subrayar que, como respuesta a la problemática presente en América Latina, ha sugerido la implementación de políticas públicas para resolver las situaciones de pobreza, daño ambiental e inadecuado uso de suelo (Vargas, 2002). De esta visión, nació el Enfoque de Gestión de Riesgo de Desastres, estableciendo una relación entre la planificación y el desarrollo; unos y otros relacionados con los objetivos de desarrollo sostenible, reconociendo que los desastres no son naturales, la vulnerabilidad tiene un rol igual de importante que los aspectos físicos y el riesgo obedece a procesos sociales que se determinan en diferentes contextos geográficos (Watanabe, 2015). En particular, aún existe dificultad por parte de los organismos de gestión para considerar la correspondencia entre peligros naturales y vulnerabilidades sociales; cayendo en una separación equivocada que no se deriva en transformaciones reales (Lampis \& Rubiano, 2012). Es necesario recalcar que el riesgo de desastres en un contexto latinoamericano se ha dado bajo la presencia de amenazas naturales, caóticas condiciones de vulnerabilidad social y desarticulación institucional y comunitaria (Maskrey, 1993). Así, la mayor cantidad de población en la región es urbana y se ha apoyado en la construcción de asentamientos informales que distan de suplir necesidades básicas (acceso a servicios) a sus habitantes (Fernández \& Rodríguez, 1996; Mansilla, 1996; Sandoval \& Sarmiento, 2018).

Dicho de otra manera, las ciudades se han convertido en escenarios de riesgo que se asocian a la degradación del ambiente y al aumento de desastres (Lavell, 1996; Metzger, 1996). Asimismo, los más vulnerables se exponen constantemente a situaciones de peligro y se enfrentan a nuevas formas de pobreza y marginalidad (Quarantelli, 1996; Park, 2000; Pizarro, 2001). Para Vargas (2002), el riesgo de desastres empeora con el crecimiento desigual y empobrecedor, siendo estas condiciones comunes en Latinoamérica y el Caribe. Entorno a la explicación de desastres hidrometereológicos en la región, el cambio climático ha sido un enfoque que resulta esclarecer los excesos ocasionados por el ser humano en un contexto global, razón que ha llevado al desarrollo de un conjunto de instrumentos técnicos de monitoreo y control de distintos fenómenos (Ferradas-Mannucci \& Torres-Guevara, 2012). Sin embargo, no se pueden atribuir todos los daños exclusivamente a este tema y menos en contexto de vulnerabilidades, donde también se concentran formas inapropiadas de ocupación del territorio bajo la mirada institucional. Alrededor de este tema, una de las organizaciones más reconocidas ha sido la Red de Estudios Sociales en Prevención de Desastres en América Latina (LA RED), creada en 1992 y siendo una referencia obligatoria de marcos teóricos y metodológicos de múltiples investigaciones (Maskrey, 1993; García-Acosta, 1996; Mansilla, 1996; Fernández, 1996; Lavell \& Franco, 1996; GarcíaAcosta, 1997; Lavell, 1997; Mansilla, 2000; Viand \& Briones, 2015). Además, se atribuye a LARED, el Sistema de Inventario de Desastres (desInventar), conjunto de datos que puede ser consultado en línea (DESINVENTAR, 2018; Zúñiga-Arias et al., 2019). Los estudios de riesgo de desastres 
requieren reconocer los lineamientos que enmarcan su comprensión. En consideración a esto, es importante reconocer la amenaza, vulnerabilidad, riesgo, inundación, gestión y ordenamiento territorial como conceptos centrales.

Para valorar el riesgo de desastres es necesario conocer la amenaza y la vulnerabilidad. La amenaza ha sido minuciosamente estudiada desde las ciencias exactas y constituye el peligro de ocurrencia de un evento de origen físico que puede causar perjuicio a la sociedad (Lavell, 1996). Estos eventos pueden afectar diferentes lugares singularmente o en combinación (fallas sísmicas, laderas, líneas costeras) (Blaikie et al., 1996). La condición de amenaza deberá ser evaluada a partir de métodos congruentes con el fenómeno a estudiar: Sismos (Saldaña et al., 2005), inundación (Rodríguez-Gaviria, 2016), movimiento en masa (Mendoza-Ramírez \& Aristizabal, 2017), incendios forestales (Muñoz-Robles et al., 2005). Al mismo tiempo, zonificar la peligrosidad por un fenómeno natural resulta ser un insumo importante para la planeación territorial, especialmente cuando se trata de comprobar la aptitud ambiental de zonas de expansión urbana o de nueva infraestructura (Cardona, 1993). La definición de vulnerabilidad se asocia con las características y capacidades de una persona o comunidad para sobrevivir y resistir el impacto de una amenaza natural (Blaikie et al., 1996). Para Quarantelli (1996) los pobres son los más vulnerables; regularmente habitan lugares susceptibles a peligros. Según Wisner et al. (2003), algunos aspectos del ambiente social se reconocen fácilmente porque la gente vive en condiciones económicas difíciles que los obligan a ocupar zonas afectadas por amenazas naturales, pero se deben reconocer también factores políticos menos obvios que influyen en la circulación de conocimiento e información y que se distribuyen en diferentes grupos sociales para crear distintas formas de discriminación. De acuerdo a Adger (2006), la medición de la vulnerabilidad debe reflejar procesos sociales, así como resultados materiales, por lo tanto, no depende exclusivamente de un número y la interpretación de un conjunto complejo de parámetros en una métrica cuantitativa reduce su impacto y oculta su complejidad.

Los estudios de amenaza y vulnerabilidad fluctúan entre enfoques de análisis que van desde las ciencias sociales a las exactas y viceversa. Sin embargo, la historia ha demostrado la gran producción de obras investigativas enmarcadas mayormente en determinar el peligro y que se remiten a modelos estructurados y muy bien abordados técnicamente. Este escenario ha propiciado la formulación de estrategias de mitigación estructurales que en muchos de los casos han sido aprovechadas por comunidades para localizar nuevas viviendas, lo cual demuestra la importancia de las ciencias humanas y sociales en el abordaje del riesgo. Así, quedó al descubierto la necesidad de fortalecer modelos de valoración con una preferencia integral y aplicada que ayuden a que los procesos de planificación se dirijan al campo específico del riesgo de desastres y representen el conocimiento oportuno para prevenir y atender en el caso de presentarse un evento (Martínez-Rubiano, 2011). Estas anotaciones fueron previstas antes de los años noventa por Kenneth Hewitt quién planteó una visión alternativa con la idea de que la amenaza no es el único agente del desastre, siendo significativo estudiar los procesos sociales que generan condiciones para que un fenómeno natural termine en desastre (García-Acosta, 2005; Ley \& Calderón, 2008). Para Hewitt, era importante la discusión acerca de que los desastres habían sido conocidos y definidos por agentes biofísicos desde una visión desde arriba, siendo necesario formular nuevos estudios desde una visión desde abajo que reflejara las condiciones reales de las personas y su funcionamiento cotidiano (Hewitt, 1996). La necesidad de articular a las victimas desde una visión desde abajo para hacer visible el espacio social de los desastres expresada por Hewitt, tuvo una importante resonancia en los años noventa con el Decenio Internacional para la Reducción de los Desastres Naturales (1990-1999). En este sentido, la producción social de vulnerabilidad deberá ser abordada con el mismo grado de importancia metodológica con la que se dedica a comprender las amenazas naturales (Blaikie et al., 1996).

En este orden, la construcción social, se ha convertido en un enfoque utilizado para explicar que el riesgo puede construirse y reproducirse al no estudiarse integralmente y sí no se toman medidas de mitigación adecuadas. Lavell (1996) señala que el riesgo, es el resultado de la interrelación de peligros y vulnerabilidades, lo cual lo convierte en una construcción social, dinámica y con características diferenciadas. El riesgo como construcción social corresponde al conocimiento de las condiciones de la amenaza (duración, frecuencia, magnitud, intensidad), en combinación con las condiciones sociales y la percepción de la población (Aneas de Castro, 2000). Podemos decir que la interpretación de construcción social de riesgo se ha asociado cotidianamente 
a la situación exclusiva de factores sociales, lo cual solo resultaría en un extremo similar al ocurrido antes de los años 60 cuando los estudios hacían referencia solamente a parámetros de ingeniería y ciencias exactas. En el caso de la gestión de riesgo constituye las políticas y acciones que tienen como propósito disminuir la afectación a comunidades y demás elementos. Según Lavell (2001), la gestión es un proceso de toma de conciencia y de decisiones para realizar acciones de mitigación de acuerdo a las características específicas de un contexto determinado. El Ordenamiento Territorial es la proyección espacial de las políticas sociales, culturales, ambientales y económicas de una sociedad (Consejo de Europa, 1983). Es importante resaltar que, la gestión de riesgo (Watanabe, 2015) y el ordenamiento territorial (Consejo de Europa, 1983; Massiris, 2002) en América Latina han hecho mayor énfasis en la amenaza que en las condiciones de tipo social (CEPAL, 2002).

A esto se suma, la debilidad institucional para conocer las amenazas, vulnerabilidades y riesgos; situación que dificulta la incorporación de criterios de mitigación en instrumentos de ordenación territorial (Rubiano-Vargas \& Ramírez-Cortés, 2009). Los anteriores conceptos se esclarecen a partir de la construcción social, enfoque que señala que el riesgo está en función de las condiciones físicas del evento natural y las características sociales presentes en la población que se expone al peligro (Hewitt, 1983). Por lo tanto, el riesgo se construye y se reproduce socialmente atendiendo a las condiciones propias del contexto, espacio y tiempo del territorio a estudiar, siendo necesario gestionarlo mediante instrumentos que permitan su ordenación. De acuerdo con esto, en Colombia existen normativas específicas en el campo de ordenamiento territorial y gestión de riesgo encaminadas a desarrollar instrumentos de gestión que orienten la planificación sostenible de los municipios. En términos generales, un Plan de Ordenamiento Territorial es el instrumento básico para desarrollar el proceso de ordenamiento del territorio municipal y se define como el conjunto de objetivos, directrices, políticas, estrategias, metas, programas, actuaciones y normas adoptadas para orientar y administrar el desarrollo físico del territorio y la utilización del suelo (República de Colombia-Ley 388, 1997). Los requerimientos para la incorporación de riesgo en términos normativos se establecen en la Ley 388/97 (República de Colombia-Ley 388, 1997) en materia de prevención y reducción de riesgos de origen natural y se debe considerar:

1. Tener en cuenta las políticas, directrices y regulaciones sobre prevención de amenazas naturales y riesgos, señalamiento y localización de las áreas de riesgo para asentamientos humanos, así como las estrategias de manejo de zonas expuestas a amenazas naturales y riesgos, que constituyen normas de superior jerarquía.

2. Componente general del Plan de Ordenamiento: determinar y ubicar en mapas las zonas que presenten alto riesgo para la localización de asentamientos humanos, por amenazas naturales o riesgos, así como las estrategias para su manejo (entendidas como los mecanismos para la reubicación de los asentamientos humanos localizados en zonas de alto riesgo y para evitar su nueva ocupación).

3. Incluir en el componente urbano del plan de ordenamiento la delimitación, en suelo urbano y de expansión urbana, de las áreas expuestas a amenazas naturales y riesgos.

4. Clasificación del suelo: la ubicación de infraestructuras para la provisión de servicios públicos domiciliarios o de las áreas de amenazas y riesgo no mitigable para la localización de asentamientos humanos, tiene restringida la posibilidad de urbanizarse.

La Ley 1523 de 2012, instituye el Sistema Nacional de Gestión del Riesgo de Desastres y dicta disposiciones para incorporar en los planes de desarrollo y de ordenamiento territorial las consideraciones sobre desarrollo seguro y sostenible, derivadas de la gestión del riesgo. En un Plan Municipal de Gestión de Riesgo, se priorizan los escenarios más frecuentes en la zona (inundaciones, movimiento en masa, incendios forestales, etc.), se caracterizan cada uno de ellos considerando elementos históricos y contextuales y se establece un componente programático para fortalecer el conocimiento del riesgo, la reducción de riesgo y el manejo del desastre. La relación que existe entre la normatividad y la organización del espacio puede ser sustentada a partir de la misma ley en la cual se plantea lo siguiente.

- La utilización del suelo por parte de sus propietarios se debe ajustar a la función social de la propiedad y permita hacer efectivos los derechos constitucionales a la vivienda, así como por la protección del medio ambiente y la prevención de desastres. 
- Función pública del urbanismo: es mejorar la seguridad de los asentamientos humanos ante los riesgos.

- Acción urbanística: la determinación de las zonas no urbanizables que presenten riesgos para la localización de asentamientos humanos, por amenazas naturales, 0 que de otra forma presenten condiciones insalubres para la vivienda.

El riesgo de desastres es determinante para establecer políticas públicas, directrices y regulaciones, señalar y localizar áreas en riesgo, definición de estrategias de manejo de áreas expuestas, definición de zonas no urbanizables, establecimiento de zonas en peligro, evaluación de zonas mitigables y no mitigables, localización de zonas críticas de recuperación y control; además es condicionante para identificar la capacidad del territorio, definir restricciones, concretar medidas de control y formular políticas, programas, planes y proyectos de reducción de vulnerabilidad social (República de Colombia-UNGRD, 2015). La Gestión de Riesgo de desastre tiene un marco conceptual determinado en el Capítulo I, artículo $1^{\circ}$ de la Ley de Gestión de Riesgos y se define como un proceso social orientado a la formulación, ejecución, seguimiento y evaluación de políticas, estrategias, planes, programas, regulaciones, instrumentos, medidas y acciones permanentes para el conocimiento y la reducción del riesgo y para el manejo de desastres, con el propósito explícito de contribuir a la seguridad, el bienestar, la calidad de vida de las personas y al desarrollo sostenible (República de Colombia - Ley 1523, 2012)

Con la necesidad de identificar las condiciones de amenaza, vulnerabilidad y riesgo a inundación y su gestión dentro de los instrumentos de ordenamiento territorial que orientan el crecimiento urbano de la ciudad, se tomó como caso de estudio Santiago de Cali, Colombia. Este trabajo se centró evaluar el riesgo por inundación mediante el método de Evaluación Sintetizada para Riesgo de Desastre con Enfoque Territorial (MESR) propuesto por la autora. La investigación se orientó especialmente al análisis de inundaciones urbanas. Sin embargo, el método podría ser aplicable a otro tipo de fenómenos (incendios, sismos, movimiento en masa, etc.). También, se realizó la zonificación utilizando sistemas de información geográfica, resultando distintos mapas que permiten visualizar espacialmente los riesgos y su distribución en el territorio; lo cual reitera la importancia de la cartografía de riesgos en la planificación y ordenación de ciudades.

\section{ESTADO DE LA CUESTIÓN}

La ciencia, ha formulado orientaciones alrededor de teorías y métodos que también son visibles desde el tema de riesgo de desastres. En cuanto a esto, se pueden identificar distintas tendencias conforme a los aportes positivistas, históricos y humanos, integrales y aplicados. El conocimiento analítico o positivista, surge de las ciencias naturales y la ingeniería, con el propósito de desarrollar procedimientos técnicos que permitan reconocer factores de tipo físico del riesgo (MartínezRubiano, 2015). Sirva de ejemplo, "El Procedimiento para Simulación Hidráulica a Partir de los Software ArcGis y HEC-RAS para eventos extremos máximos" en la cuenca del río Sagua la Chica en Cuba (López-Rodríguez, 2017). La particularidad funcional de estos estudios es reconocida como paradigma fiscalista por no tener en cuenta la sociedad expuesta y centrarse en que los desastres son productos de fenómenos extremos que se desarrollan por asociación natural (Hewitt, 1983). En el caso de la tendencia histórica y humana, se sustenta en la antropología, sociología y psicología, que indagan el papel de la sociedad ante peligros y desastres, considerando la percepción y actitudes conforme a la experiencia del sujeto. Estos estudios admiten la interrelación, la valoración de la diferencia cultural y la responsabilidad (Martínez-Rubiano, 2015). Así que, la forma de comprender y afrontar las situaciones de riesgo dependen en gran medida de la cosmovisión de las persona en relación con la capacidad de organización, el conjunto de valores, patrones familiares y la transformación de actividades humanas en función de esquemas globales que rigen sistemas políticos y económicos (Quarantelli, 1996).

Para ilustrar mejor, en el artículo "Percepción del Riesgo y Estrategias de Afrontamiento en Población Expuesta y no Expuesta al Riesgo de Inundación", se encontró que las personas más propensas a sufrir inundaciones tienen mayor temor a ser afectados y desarrollan estrategias para afrontar el evento (Navarro et al., 2016). La tendencia integral, es el conocimiento aplicado en el cual las amenazas y las condiciones sociales se estudian para entender su interrelación y reconocer las condiciones que deben ser transformadas mediante la gestión de riesgo (MartínezRubiano, 2015). En este sentido, las metodologías para evaluar la amenaza han sido abordadas tradicionalmente y han probado numerosas investigaciones para conocer los peligros aportando 
a los organismos de control que hacen frente al desastre. El desarrollo de nuevas alternativas para estudiar el riesgo ha estado influenciado por los estudios integrales y aplicados. Vale la pena precisar que, entre sus principales aportes se considera el concepto de vulnerabilidad (Maskrey, 1993). En otras palabras, las realidades actuales obligan a interpretar los riesgos como un proceso socialmente construido. En cuanto a esto, en el Decenio Internacional para la Reducción de los Desastres, se generaron muchas investigaciones en esta materia. Entre las conclusiones derivadas de estos hallazgos se encontró que, la mayoría de los desastres tenían una relación intrínseca con el crecimiento demográfico, la urbanización, la degradación ambiental y al mismo tiempo, se incrementaban con las desigualdades socioeconómicas (García-Acosta, 2005). En efecto, la dinámica entre la sociedad y la naturaleza produce condiciones complejas que alteran el entorno y esos procesos, reducen o intensifican los desastres (Alcaldía Municipal de Santiago de Cali, 2014). Para García-Acosta (2005), la construcción social de los riesgos relacionada con las condiciones de vulnerabilidad y desigualdad, pueden producir nuevas amenazas que se asocian con la acumulación material de los riesgos.

Así, por ejemplo, muchas personas viven en condiciones socioeconómicas precarias y esto los obliga a ocupar zonas amenazadas constantemente (Wisner et al., 2003). Sin duda, la predictibilidad del desastre estaría comprobada a partir del peligro y al exponerse en espacios no aptos a causa de la vulnerabilidad (Cardona, 2001). Para García-Acosta (2005), la construcción social del riesgo es un proceso que necesita ser estudiado desde una perspectiva histórica debido a que el riesgo se desarrolla, se acumula y puede transformarse en el tiempo. De acuerdo a Narváez et al. (2009), el fundamento de este enfoque radica en que de manera natural se presentan eventos físicos que pueden ser generados por dinámicas ambientales, pero el significado de amenaza 0 peligro está mediada por la intervención humana. Por consiguiente, el nivel de riesgo se determina además del fenómeno por el estado de exposición y de vulnerabilidad social. En esta medida, ocurrencias menores pueden causar más daño ante mayor exposición y vulnerabilidad. Bajo estos argumentos, el enfoque de construcción social del riesgo sostiene que las afectaciones no están en función exclusiva del evento físico y su intensidad (Hewitt, 1983). En este sentido, es un enfoque que explica que el riesgo puede construirse y reproducirse al no estudiarse de forma integral y sin determinar las medidas adecuadas para evitarlo y reducirlo.

También indica que el riesgo es producto de un proceso social e histórico que puede llegar a manifestarse en un desastre y expresar una convivencia vulnerable entre diferentes grupos sociales y el ambiente (Herzer, 2011). Además, es multicausal y las condiciones de marginalidad, pobreza, densidad de población, falta de control territorial y percepción humana pueden empeorar la condición o influir en la producción de nuevos escenarios de riesgo (Rodríguez-Esteves, 2007). Por lo tanto, comprender la amenaza, las características socioeconómicas, vulnerabilidades y procesos de adaptación en un contexto espacial y temporal, son fundamentales para el análisis de construcción social de riesgo (Rodríguez-Esteves, 2007). Para Lavell (1996), el riesgo es el resultado de la interrelación de peligros y vulnerabilidades. Por consiguiente, es una construcción social, cambiante y con tipologías diferenciadas. El valorar el riesgo de desastre desde el enfoque de construcción social, requiere estimar la vulnerabilidad con la misma rigurosidad e importancia con la que se han estudiado las amenazas naturales. Es decir, el riesgo es una composición entre la vulnerabilidad y el peligro sin existir una sin la otra (Blaikie et al., 1996). Tal como lo menciona Narváez et al. (2009), los factores de riesgo y su construcción social dependen de amenazas físicas naturales y vulnerabilidades que se desarrollan en magnitudes, tiempos y contextos diferenciados. La construcción social del riesgo se sustenta en dos procesos importantes:

Un evento físico particular (manifestación del ambiente), o conjunto de ellos, con potencialidad para causar daños y pérdidas adquiere la connotación de peligrosidad. Esto sucede cuando elementos socioeconómicos son expuestos en condiciones de vulnerabilidad en áreas de potencial afectación o presencia de los fenómenos físicos peligrosos. Nuevos eventos físicos son generados por intervención humana en la transformación del ambiente natural (eventos socio-naturales), o por efecto directo del manejo, producción y/o distribución de materiales peligrosos (eventos antrópicos).

(Narváez et al., 2009, p.11)

Es decir, el riesgo es construido socialmente cuando la población se expone a situaciones de peligro debido a la intervención que realiza en un territorio. Esto generalmente va acompañado 
de vulnerabilidades comunitarias que favorecen tomar la decisión de ocupar zonas amenazadas por inundaciones u otro tipo de evento. Vale la pena precisar que desde la construcción social del riesgo es fundamental el concepto de vulnerabilidad. Por lo tanto, las evaluaciones se han centrado en la vulnerabilidad relacionada con la percepción humana y vulnerabilidad asociada a desigualdad social (García-Acosta, 2005). Mientras que los estudios integrales han abordado la temática desde la construcción social implícita. A causa de esto, muchos investigadores se han preocupado por fortalecer este enfoque desde trabajos teóricos y aplicados -aunque muchos no lo refieran explícitamente, su abordaje es notorio. Sirva de ejemplo Meyer et al. (2008) con la utilización de criterios económicos, sociales y ambientales para el río Mulde en Sajonia, Alemania. También, Shever et al. (2011) integrando dimensiones económicas, sociales y ecológicas para analizar el riesgo por inundación en Alemania; Dang et al. (2011) aplicando medidas económicas sociales y ambientales en la valoración del riesgo por inundación en el área del río Day en Vietnam; Asimismo, Siddayao et al. (2014) incorporaron la densidad de población en el análisis de riesgo en el municipio de Enrile en la provincia de Cagayan, norte de Filipinas; Shivaprasad-Sharma et al. (2018) clasificando el riesgo de inundación a partir de datos de vulnerabilidad física, social, económica y medio ambiental en la cuenca del río Kopili, Assam, India.

Para Latinoamérica, se identificaron cuatro modelos usados ampliamente: Vulnerabilidad Global, estructurado a partir de parámetros individuales en relación con la amenaza y a las condiciones sociales (Wilches-Chaux, 1993); Modelo Presión Liberación (PAR), correspondiente al proceso de 'presión' que integra causas de fondo, presiones dinámicas y condiciones inseguras de la población expuesta al desastre y 'liberación', fundamentada en las medidas de mitigación (Blaikie et al., 1996); Vulnerabilidad del Lugar, se compone de condiciones biofísicas y sociales que deben ser tenidas en cuenta para el estudio del riesgo (Cutter, 1996); Riesgo Holístico, se constituye de variables físicas y sociales y su aplicación se ha dado principalmente en el análisis de sismos (Cardona, 2001). Esta investigación, se enmarca dentro de la construcción social del riesgo, la cual sostiene que los riesgos son socialmente construidos y conllevan a diferentes experiencias en tiempo y espacio (García-Acosta, 2005). Esto para explicar que los patrones de crecimiento poblacional han incidido en la organización espacial de la ciudad y el riesgo a inundaciones. Conforme a esta línea, se pretende abordar el riesgo desde una definición teórica incluida en los conceptos de amenaza, vulnerabilidad, riesgo, gestión y ordenamiento territorial.

- Amenaza. Peligro de inundación debido a condiciones naturales y estructurales presentes en la ciudad.

- Vulnerabilidad. Conjunto de desigualdades históricas, sociales, económicas y territoriales que reflejan condiciones de fragilidad para enfrentar una inundación y obligan a la población urbana a ocupar zonas no aptas.

- Riesgo. La interacción entre las condiciones de amenazas o peligro por inundación y la vulnerabilidad.

- Gestión de riesgo. Conjunto de políticas y acciones que impulsan la disminución de muertes y daños materiales.

- $\quad$ Ordenamiento del territorio. Elemento de apoyo a la gestión de riesgo que orienta el crecimiento urbano sostenible mediante instrumentos de planificación.

El desarrollo de este trabajo supone una visión tanto física y social del fenómeno a estudiar, por lo tanto, se siguió un método propio formulado a partir de cuatro modelos que conciben ambas interpretaciones: Vulnerabilidad Global (Wilches Chaux, 1993); Modelo Presión Liberación (PAR) (Blaikie et al., 1996); Riesgo Holístico (Cardona, 2001). Vulnerabilidad del Lugar (Cutter et al., 2003). Los métodos propuestos por estos autores tienen en común la lectura de las ciencias físicas y sociales presentando distintas dimensiones de análisis, lo cual orienta la visión de construcción social del riesgo que nos interesa. Se retoma los planteamientos expresados por los autores y de manera general en la Tabla 1 se presentan las dimensiones.

Así las cosas, es importante centrarse en las desventajas identificadas en los modelos estudiados como oportunidad para la elaboración de un modelo con enfoque de ordenamiento territorial que, además de los componentes teóricos y/o metodológicos se apoyen en la representación espacial de fenómenos de amenaza, vulnerabilidad y riesgo, a una escala en el territorio que permita su evaluación y mitigación dentro de los procesos de planificación. 


\begin{tabular}{llll} 
Vulnerabilidad Global & Presión-Liberación & Vulnerabilidad del Lugar & Riesgo Holístico \\
\hline $\begin{array}{l}\text { Vulnerabilidad Global. } \\
\text { Aborda distintos tipos }\end{array}$ & Modelo de Presión y & La utilización de & El método es específico \\
de vulnerabilidad. & siglas en inglés). Modelos & indicadores precisos & a la consideración de \\
En sus inicios, tenía & generales y específicos & para el análisis territorial & sismos. Sin embargo, es \\
pocos elementos que & por fenómenos de & y construcción de & utilizado, es fácil de \\
orientaran su medición, & desastres. Aunque & cartografía. Aunque el & interpretar y en los \\
lo que favorecía análisis & presenta gran dificultad & modelo es ampliamente & últimos años ha sido \\
cualitativos. Sin embargo, & de interpretación de cada & seguido, no se percibe & adaptado a otros \\
nuevos investigadores & uno de sus componentes, & evolución en las & fenómenos de riesgo. \\
han enriquecido el & es el único que propone & técnicas y en los análisis & Sin embargo, incorpora \\
método, reforzando & un componente de & propuestos en nuevos & un componente de \\
investigaciones de todo & identificación (Presión) & trabajos. & exposición que puede \\
tipo. & y otros de mitigación & & ser determinado en la \\
& (Liberación). & & condición de amenaza. \\
\hline (Wilches-Chaux, 1993) & (Blaikie et al., 1996) & (Cutter et al., 2003) & (Cardona, 2001) \\
\hline
\end{tabular}

Tabla 1. Métodos integrados Fuente: Autora, 2021.

Además de la ubicación cartográfica, es indispensable el abordaje cualitativo en el componente explicativo, este último dará cuenta de las realidades construidas socialmente y que han influido y seguirán influyendo en un contexto de presente y futuro. En tanto, la revisión de los modelos anteriormente citados permitió pensar un método que respondiera a la diferencia entre procesos de riesgo, la utilización de cartografía de riesgo, además de servir como insumo para la mitigación y la ocupación adecuada del suelo. Estas consideraciones estimularon desechar lo siguiente.

- Se desechó el empleo de parámetros relacionados con la atención a la emergencia (camas, hospitales, respiradores, sillas, etc.), esto obedeció a que este trabajo se centra en conocer el riesgo (amenazas, vulnerabilidades y elementos expuestos). Por lo tanto, se considera que esos parámetros obedecen al manejo del desastre y sería más pertinente en modelos de atención a la emergencia.

- Se desechó la utilización de un método multivariante para priorizar indicadores (como el utilizado por Cutter). Esto debido a que las condiciones de vulnerabilidad histórica, social, económica y territorial, obedecen a insumos de distinta naturaleza que necesitan ser identificados, para posteriormente explicados y mitigados a partir de acciones concretas; siendo más útil para el ordenamiento del territorio y la planificación urbana (Adger, 2006). Por consiguiente, intentar reducir componentes sociales mediante métodos estadísticos puede implicar demeritar las necesidades de la población dificultando el establecimiento de programas de gestión.

- Se desecharon variables que no pudieran ser integradas a herramientas de análisis geoespacial.

En el nuevo modelo se incorporó la dimensión de vulnerabilidad histórica, las variables migración y etnia siendo utilizadas en trabajos previos como parte de las características socioeconómicas de la población. Sin embargo, en esta investigación se alude a los migrantes más vulnerables que viajan de departamentos, estados o países más pobres y/o aquellos que no cuentan con datos para visibilizar su situación; población que generalmente sale de su lugar de origen buscando mejores oportunidades de vida, con la intención de ayudar a familiares a subsistir y con historias de vida desoladoras. Estas condiciones migratorias pueden obligarlos a ubicarse en zonas, ciudades, estados y hasta países específicos (ejemplo: población africana en Europa). La etnia para el caso de Colombia hace referencia a los negros y a los indígenas considerados minorías, grupos que han arrastrado un legado histórico de esclavitud y colonización que incluso se mantiene en la actualidad. Pongamos, por ejemplo, la tasa de pobreza en México y la presencia racial y encontramos que coinciden en los estados de Chiapas, Guerrero, Oaxaca y Veracruz. Sin embargo, en otros contextos pueden variar las desigualdades (judíos en el Alemania Nazi, gitanos en España, etc.). También, se añadió la vulnerabilidad territorial como un componente que reúne condiciones de acceso a estructuras funcionales de la ciudad. La dimensión de vulnerabilidad territorial puede demostrar segregación espacial, siendo esto muy propio de los centros urbanos en América Latina, donde existe una amplia creación de asentamientos informales 
y una institucionalidad que obedece más a condiciones impuestas por el mercado del suelo al que no todos pueden acceder. Asimismo, se agregó un componente de elementos expuestos de manera externa a los aspectos relacionados con la vulnerabilidad, esto debido a que se pueden determinar la exposición de personas, estructuras físicas, sociales, etc.; desde que se identifica la situación de peligro y se reconoce que todos los insumos que hacen parte del modelo dependen de las características propias de cada territorio.

Vale la pena resaltar que, este método tiene un enfoque de ordenamiento territorial porque permite la inclusión de cartografía de riesgo a instrumentos que pueden hacer posible reducir las condiciones de amenaza y vulnerabilidad, posibilitando la gestión a favor de la planificación física y sus usos, el equilibrio socioeconómico y la reducción de desigualdad en un territorio determinado por un tiempo y un espacio particular (Urteaga, 2011).

Por tanto, la aplicación metodológica en relación al riesgo de desastres debe ser encaminada indiscutiblemente en términos de ordenamiento territorial, en tanto que, llevan a que las condiciones de amenaza y vulnerabilidad puedan ser medidas rigurosamente, para posteriormente ser equiparadas en un solo componente que corresponderá al riesgo de desastres. Esto se constituye en un horizonte de uso de suelo planificado a favor del desarrollo social y disminución de riesgo de desastres. Conviene subrayar que, las consideraciones desarrolladas por la autora adquieren un enfoque operacional y corresponden en su aplicación metodológica.

Amenaza. Valorada a partir de la probabilidad de inundación de la ciudad.

Vulnerabilidad sintetizada. Estimada desde indicadores extremos de desigualdad social y accesibilidad, es considerada como una dimensión de síntesis porque su resultado final reúne varias condiciones de análisis. En este caso se tienen en cuenta cuatro dimensiones de análisis:

- Vulnerabilidad histórica.

- Vulnerabilidad social.

- Vulnerabilidad económica.

- Vulnerabilidad territorial.

Riesgo. Evaluada desde la combinación de las zonificaciones de amenaza y vulnerabilidad.

Elementos expuestos. Correspondiente a todos los elementos que se encuentren expuestos en condiciones de peligro, vulnerabilidad o riesgo.

Lo dicho hasta aquí supone que, se deberá hacer un diagnóstico de amenaza, vulnerabilidad y riesgo. Interpretación que se facilita con el uso de herramientas de posicionamiento global y elaboración de cartografía. Sin embargo, otro aspecto importante es el proceso de gestión a favor de la reducción de las afectaciones por inundaciones. Situación que favorece orientaciones apropiadas para el uso de suelo a partir de instrumentos de planificación como lo son los Planes de Ordenamiento Territorial y de Gestión de Riesgo. Así las cosas, es importante anotar que uno de los elementos que interviene en la construcción social del riesgo es el «territorio». Massiris (2002), lo aborda como un espacio humanizado, concreto, relacional, resultado del movimiento y la interacción de los fenómenos sociales incluidos los soportes físicos en los cuales se desarrollan. El territorio se convierte en una condición integradora de las manifestaciones físicas y sociales que pueden sustraerse de enfrentar una situación de desastre.

El territorio es el espacio geográfico donde un estado ejerce su soberanía y realiza planes y programas de desarrollo a escala subregional y local (Poinsot, 1992). Desde una óptica sistémica se concibe como un espacio humanizado, concreto, relacional, resultado del movimiento y la interacción de los fenómenos sociales incluidos los soportes físicos en los cuales se desarrolla (Massiris, 1999). En el mismo, se deben ejercer acciones político-administrativas que se sustentan en el ordenamiento territorial, el cual es entendido como una política de estado y proceso político, técnico, administrativo planificado y concertado, con el que se pretende configurar, en el largo plazo una determinada organización del uso, la ocupación del territorio y orientar su transformación (Massiris, 2002).

Con esto se puede decir que, el territorio que nos interesa estudiar corresponde a una de las ciudades más importantes de Colombia, la misma que presenta unas condiciones climáticas que determinan la presencia de fenómenos naturales adversos y, además, muestra unas características 
sociales que ayudan a construir escenarios de riesgos. Finalmente, entender el riesgo desde la construcción social permite explicar que los riesgos son socialmente construidos, son mucho más interesantes si se sigue un enfoque integral que permitirá descubrir que esa construcción depende también de las condiciones físicas y que todos estos elementos reunidos deben ser abordados desde los estudios urbanos, siendo el ordenamiento territorial una concepción muy importante en esta línea. Bajo esta consideración, la única intención será aportar a planificar el crecimiento de ciudades más seguras y personas menos vulnerables.

\section{MÉTODO}

\section{Zona de estudio}

Santiago de Cali se encuentra al sur occidente de Colombia, es la capital del Valle del Cauca y la tercera ciudad más importante del país. En la actualidad cuenta con una población urbana de 2.172.527 (República de Colombia-DANE, 2018). La estructura geomorfológica se define a partir del valle aluvial del río Cauca al oriente y un área montañosa al occidente. La conformación hídrica es determinada a partir de los ríos Cali, Meléndez, Aguacatal, Lili, Pance y Cañaveralejo que cruzan la ciudad para nutrir la corriente principal del Cauca. Del sistema hídrico también hacen parte 48 humedades, siendo las lagunas el Pondaje y Charco Azul las más importantes (Alcaldía de Santiago de Cali-DAGMA, 2010; Alcaldía Municipal de Santiago de Cali, 2014). La organización espacial en la ciudad se integra principalmente de 22 comunas y 337 barrios, además de un área de expansión (ver Figura 1).
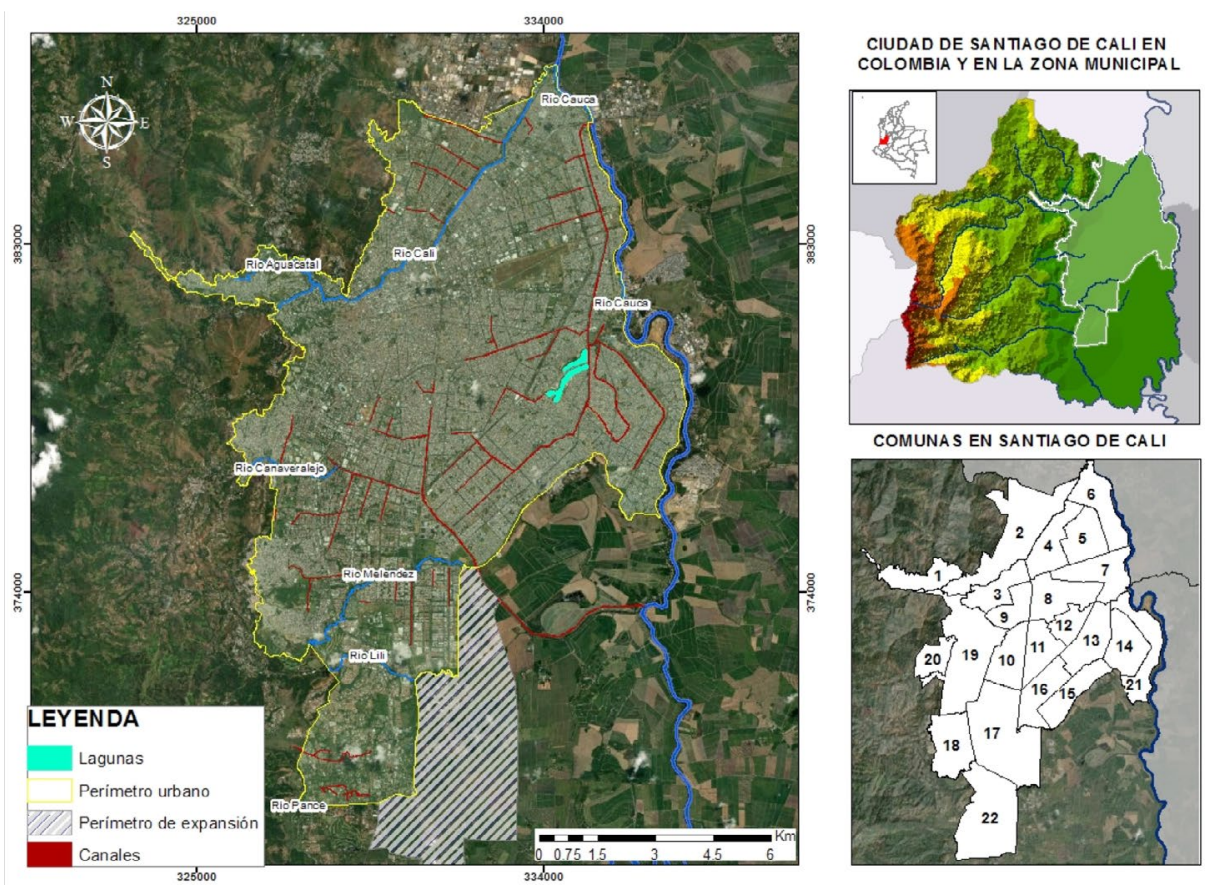

Figura 1. Localización de la zona de estudio Fuente: Autora, 2021.



El desarrollo urbano de la ciudad ha favorecido el asentamiento de viviendas hacia la zona de montaña y sobre el valle aluvial del río Cauca, en estos lugares históricamente se han reportado desastres asociados con movimiento en masa e inundaciones (Jiménez, 2005; Sevillano-Rodríguez \& Bravo Peña, 2018). En el proceso de expansión física de la ciudad la dinámica de los siete ríos (Cauca, Cali, Aguacatal, Cañaveralejo Lili, Meléndez y Pance), han estado muy presentes en su configuración urbana. En 1960 se inició la construcción de diques, canales y estaciones de bombeo dentro del Plan del Proyecto Aguablanca; en la medida que se fueron estableciendo las obras comenzaron a instalarse asentamientos formales e informales (Jiménez, 2005). La presencia de inundaciones en Cali ha tenido origen fluvial y pluvial. La primera por el desbordamiento de los ríos y la segunda debido a la insuficiencia de canales que atraviesan varios sectores de la ciudad y que en algunos casos canalizan fuentes hídricas (es el caso del canal Interceptor Sur). Por consiguiente, desde 1970 en todos los años han ocurrido inundaciones (excepto en 1991, año de escasez de lluvias) (Alcaldía Municipal de Santiago de Cali, 2014). Según Sevillano-Rodríguez et al. (2020) entre 1986-2018 se reportaron 766 inundaciones en 186 barrios de la ciudad. 


\section{Materiales}

En este trabajo, se recurrió a zonificaciones previas de peligro por inundación y vulnerabilidad (indicadores históricos, socioeconómicos y de funcionalidad territorial). Los princípiales insumos para la aplicación MESR, fueron los siguientes:

\section{Evaluación de Amenaza o peligro}

- Zonificación de inundación con periodo de retorno de 1.1 años: Información en formato raster, obtenida mediante técnicas de teledetección y análisis multicriterio realizadas previamente a partir de imágenes Sentinel 1, suministradas por el portal https://www. asf.alaska.edu/ (NASA, 2018; Sevillano-Rodríguez et al., 2020; Sevillano-Rodríguez, 2020).

\section{Evaluación de Vulnerabilidad}

- Zonificación de vulnerabilidad: Obtenida previamente por la autora mediante clasificación de información histórica, socioeconómica y de funcionalidad territorial (República de Colombia-DANE, 2005; Alcaldía Municipal de Santiago de Cali - IDESC, 2014; República de Colombia - DANE, 2018 DESINVENTAR, 2018; República de Colombia-UNGRD, 2018; Alcaldía de Santiago de Cali, 2018).

Datos de elementos expuestos

- Datos de elementos expuestos al riesgo: bases cartográficas de asentamientos informales, barrios, vías, instituciones educativas, centros de salud, estaciones de bombeo, plantas de agua potable, red principal de gas y torres de comunicación, (Alcaldía Municipal de Santiago de Cali - IDESC, 2014).

\section{Técnicas de análisis}

El procedimiento seguido corresponde al Método de Evaluación Sintetizado para Riesgo de Desastres con Enfoque de Ordenamiento Territorial (MESR). Bajo este parámetro el riesgo, es la combinación de amenaza y de vulnerabilidad a partir de lo cual se determinan los escenarios con mayor grado de exposición ante el fenómeno. Esta metodología ha sido diseñada por la autora con la intención de identificar el riesgo de desastre desde una concepción territorial y apoyada en sistemas de información geográfica.

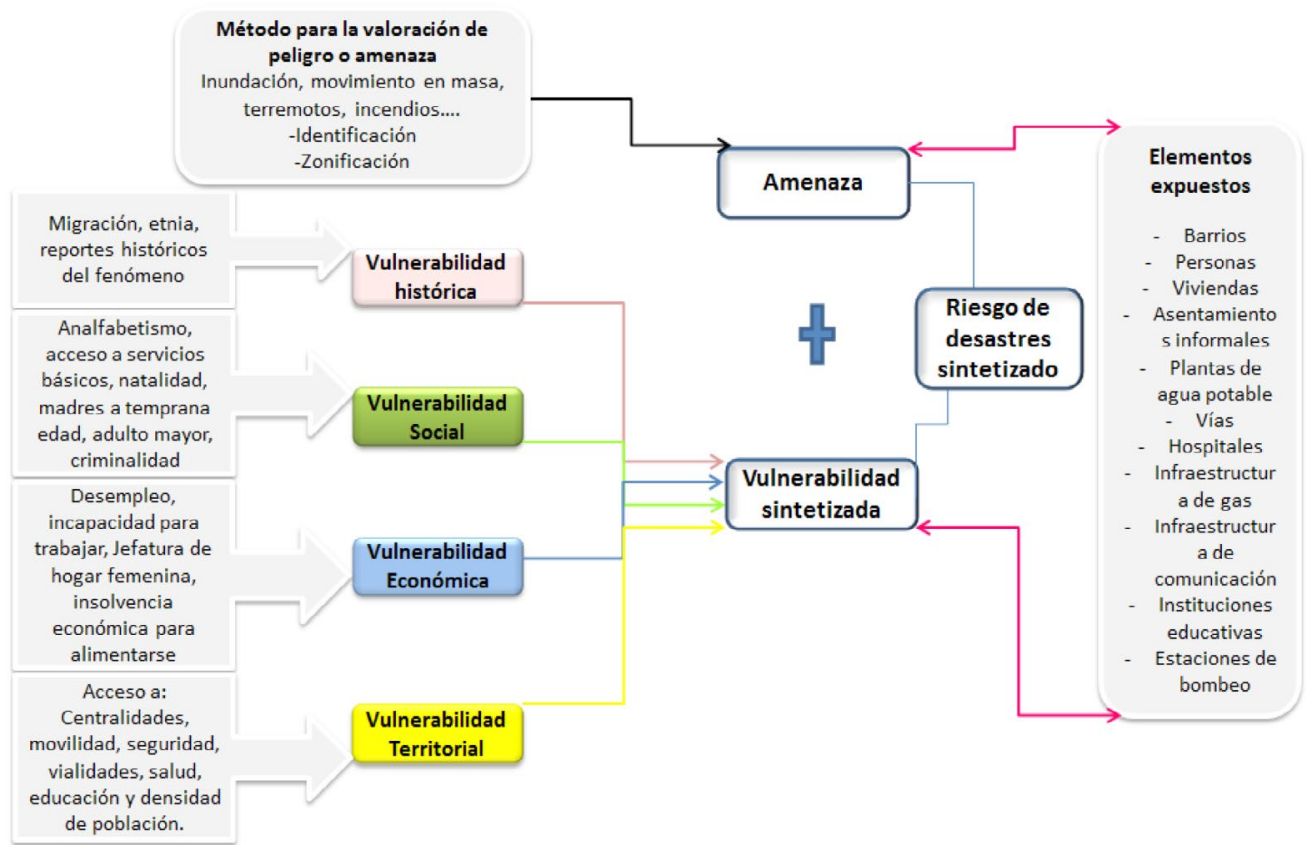

Figura 2. Método de Evaluación Sintetizado para Riesgo de Desastres con Enfoque de Ordenamiento Territorial (MESR) Fuente: Autora, 2021.

En la Figura 2, se muestra el MESR. En el caso, el riesgo de desastres corresponderá a la suma de la zonificación del peligro y la vulnerabilidad sintetizada (histórica, social, económica y territorial). En consecuencia, se pueden establecer los elementos expuestos en cualquier 
condición de riesgo (elementos expuestos de amenaza, vulnerabilidad o riesgo). En este caso, se utiliza la amenaza de inundación y una serie de factores sociales y territoriales que ocasionan la vulnerabilidad sintetizada. De la combinación de estos componentes (amenaza-vulnerabilidad) resultara el riesgo.

\section{Procesamiento de información}

Para procesar los datos se utilizó el software ArcMap. La información se generalizó en el sistema de coordenadas: Colombia - MAGNA_Cali_Valle_del_Cauca_2009. El punto de inicio correspondió al conocimiento de la cartografía de amenaza de inundación y su zonificación (alto, medio, bajo) para la ciudad de Cali, Colombia. Esta información proviene de datos trabajados previamente por la autora (Sevillano-Rodríguez et al., 2020). Seguidamente, se consideró la fragilidad social de la población representada en la vulnerabilidad sintetizada.

Riesgo de desastre sintetizado, es la suma entre la zonificación de amenaza y la zonificación de vulnerabilidad para dar resultado al riesgo. En la Tabla 2, la matriz de peligro y vulnerabilidad que permitió realizar la operación.

\begin{tabular}{lcc}
$\begin{array}{c}\text { Amenaza } \\
\text { (Condiciones Físicas) }\end{array}$ & $\begin{array}{c}\text { Vulnerabilidad } \\
\text { (Condiciones históricas, } \\
\text { socioeconómicas y territoriales) }\end{array}$ & $\begin{array}{c}\text { Riesgo } \\
\text { (Amenaza + Vulnerabilidad) }\end{array}$ \\
\hline Baja (1) & Baja (1) & Bajo (2) \\
\hline Baja (1) & Media (2) & Medio (3) \\
\hline Baja (1) & Alta (3) & Medio (4) \\
\hline & Baja (1) & Medio (3) \\
\hline Media (2) & Media (2) & Medio (4) \\
\hline Media (2) & Alta (3) & Alto (5) \\
\hline Media (2) & & Medio (4) \\
\hline Alta (3) & Baja (1) & Alto (5) \\
\hline Alta (3) & Media (2) & Alto (6) \\
\hline Alta (3) & Alta (3) & \\
\hline
\end{tabular}

Tabla 2. Matriz de peligro y vulnerabilidad Fuente: Autora, 2021.

Los elementos expuestos en el caso de la ciudad de Cali correspondieron a la cantidad de barrios y personas en riesgo alto y a los servicios urbanos que podrían alterarse en una situación de riesgo alto y medio (plantas de tratamiento de agua potable, vías primarias y secundarias, torres de comunicación, hospitales, redes principales de gas, instituciones educativas y estaciones de bombeo).

\section{RESULTADOS}

Es relevante reconocer la importancia de la utilización de técnicas semi-automatizadas que se aproximen a condiciones reales de ocupación del territorio a favor de ciudades más seguras y menos vulnerables ante eventos de desastres (Masgrau, 2004; Olcina-Cantos \& Díez-Herrero, 2017). En este sentido, todo el proceso de resultados estuvo apoyado por sistemas de información geográfica, facilitando la elaboración de mapas mediante valoración de variables y superposiciones que permitieron llevar a cabo el modelo (MESR) concebido previamente. En vista de que la propuesta metodológica planteó la necesidad de basarse en la construcción social de riesgo que, implica reconocer las condiciones de amenaza y vulnerabilidad del territorio, se obtuvieron resultados en torno a la distribución individual (amenaza, vulnerabilidad) y a la combinación general de factores que indicaron el riesgo sintetizado. En la Figura 3(A), la zonificación de amenaza o peligro de inundación para un periodo de retorno de 1.1 años (tomando como base las precipitaciones asociadas a la inundación estudiada). En la Figura 3(B), la localización de 37 barrios expuestos en amenaza alta y en la Figura 3(C), 96 barrios en peligro medio de inundación.

Según Acosta-Medina (2013), la desigualdad en Colombia se presenta en todos los niveles de la vida nacional y es muy evidente en la población más vulnerable. En este sentido, Santiago de Cali es un claro ejemplo. 


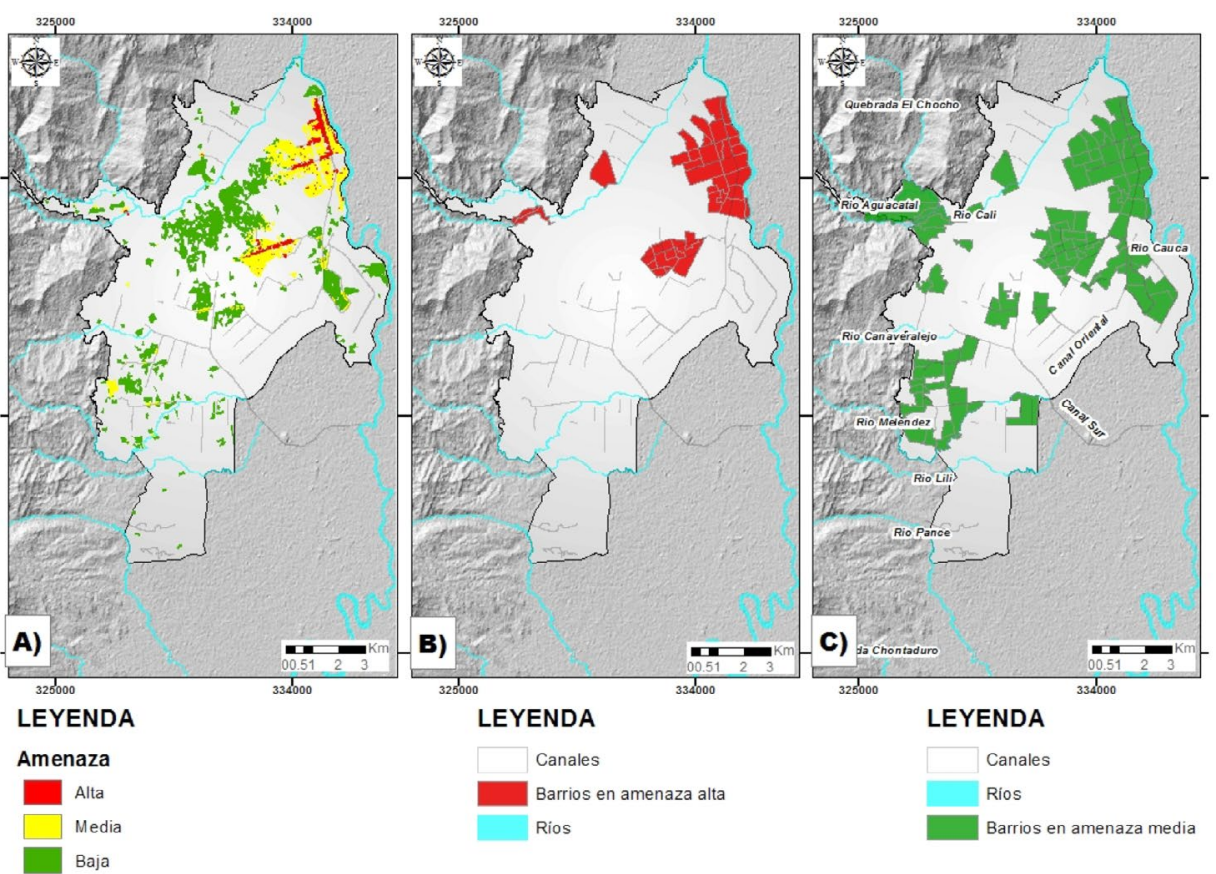

Figura 3. Amenaza por inundación y barrios expuestos en peligro alto y medio Fuente: Autora, 2021, con base en Sevillano-Rodríguez et al., 2020

En la Figura 4(A), la vulnerabilidad sintetizada clasificada en tres niveles (alta, media, baja). En la Figura 4(B), la ubicación de 144 asentamientos ilegales de los cuales 16 está en proceso de desalojo. En la Figura 4(C), los 17 barrios más vulnerables y en peligro de inundación. En la Figura 5 , asentamientos informales en la comuna 6 (al oriente de la ciudad) y edificios en la comuna 2 (al oriente de la ciudad).
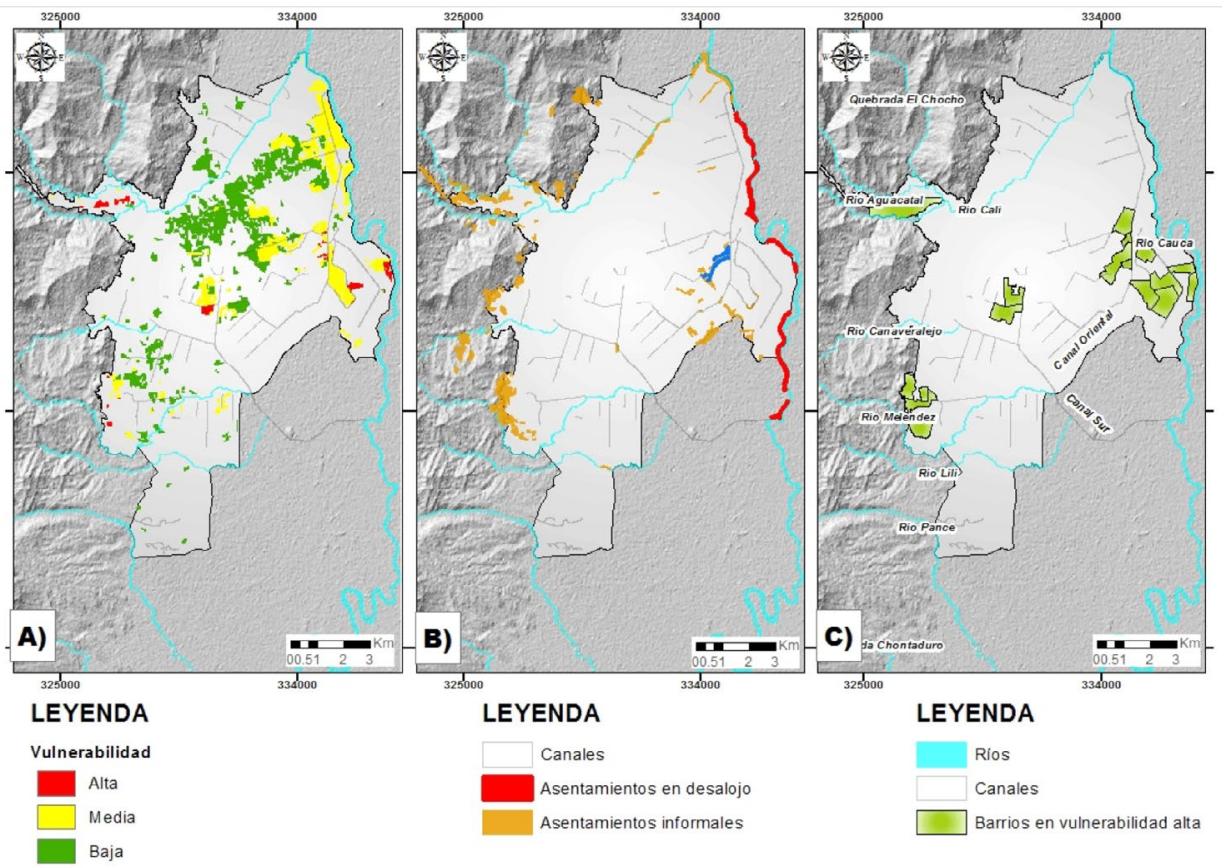

\section{LEYENDA}

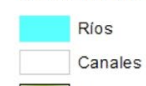

Canales

Asentamientos informales

Figura 4. Vulnerabilidad social ante inundación, asentamientos informales y barrios con mayor fragilidad Fuente: Autora, 2021, con base en Sevillano-Rodríguez et al., 2020. 


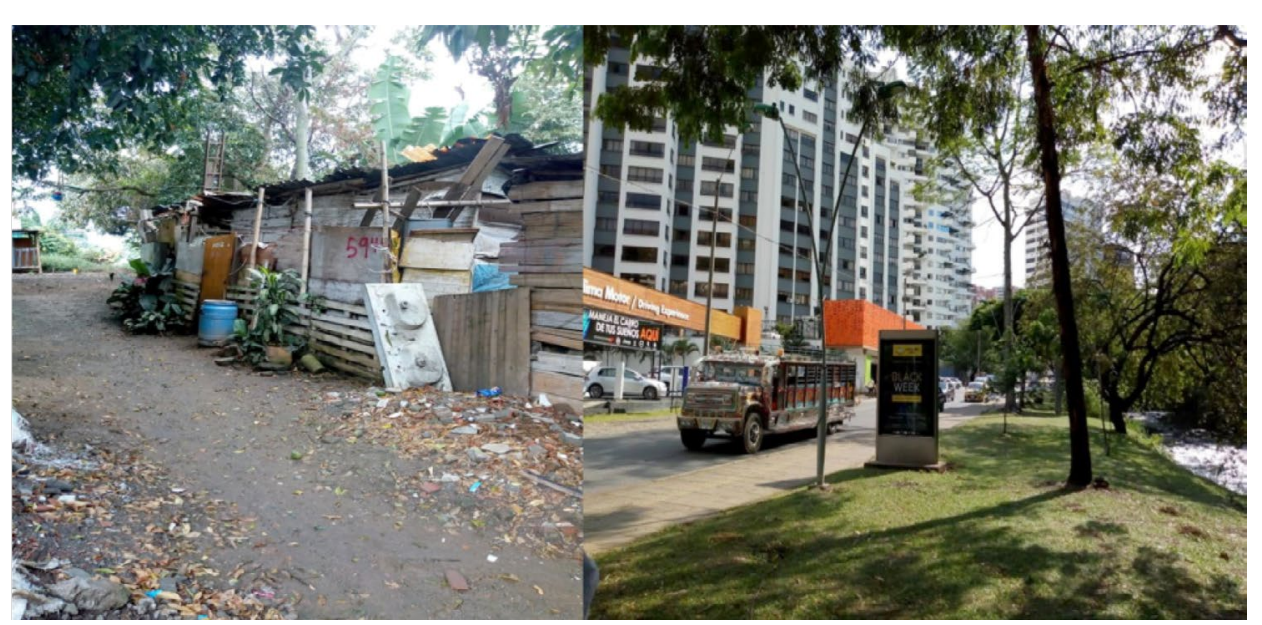

Figura 5. Asentamiento informal en comuna 6 Cali, Colombia; edificios en comuna 2 Cali, Colombia Fuente: Autora, 2020, durante recorrido de campo.

Integración del modelo final. Clasificada la amenaza y la vulnerabilidad sintetizada, se sumaron los parámetros siguiendo la matriz de peligro y vulnerabilidad (ver Tabla 2). El resultado de la operación muestra en la Figura 6, el riesgo por inundación. Esta cartografía se conformó por variables físicas (zonificación de inundación) y de vulnerabilidad (histórica, social, económica y territorial). Por lo tanto, se localizaron espacialmente los lugares y se clasificaron en tres categorías de riesgo: Alto $118 \mathrm{Ha} \mathrm{(6 \% ),} \mathrm{medio} 749 \mathrm{Ha} \mathrm{(38 \% )} \mathrm{y} \mathrm{bajo} 1115 \mathrm{Ha} \mathrm{(56 \% ).} \mathrm{Los} \mathrm{puntos} \mathrm{de} \mathrm{mayor}$ afectación se ubicaron al Este junto al canal Oriental, el río Cauca, las lagunas Charco Azul y el Pondaje; al Occidente cerca de los ríos Cali y Aguacatal; y en el Centro, cerca del Canal Interceptor Sur. Es necesario recalcar que, un gran número de viviendas localizadas en estos lugares se construyeron inicialmente bajo condiciones de ilegalidad (Jiménez, 2005).

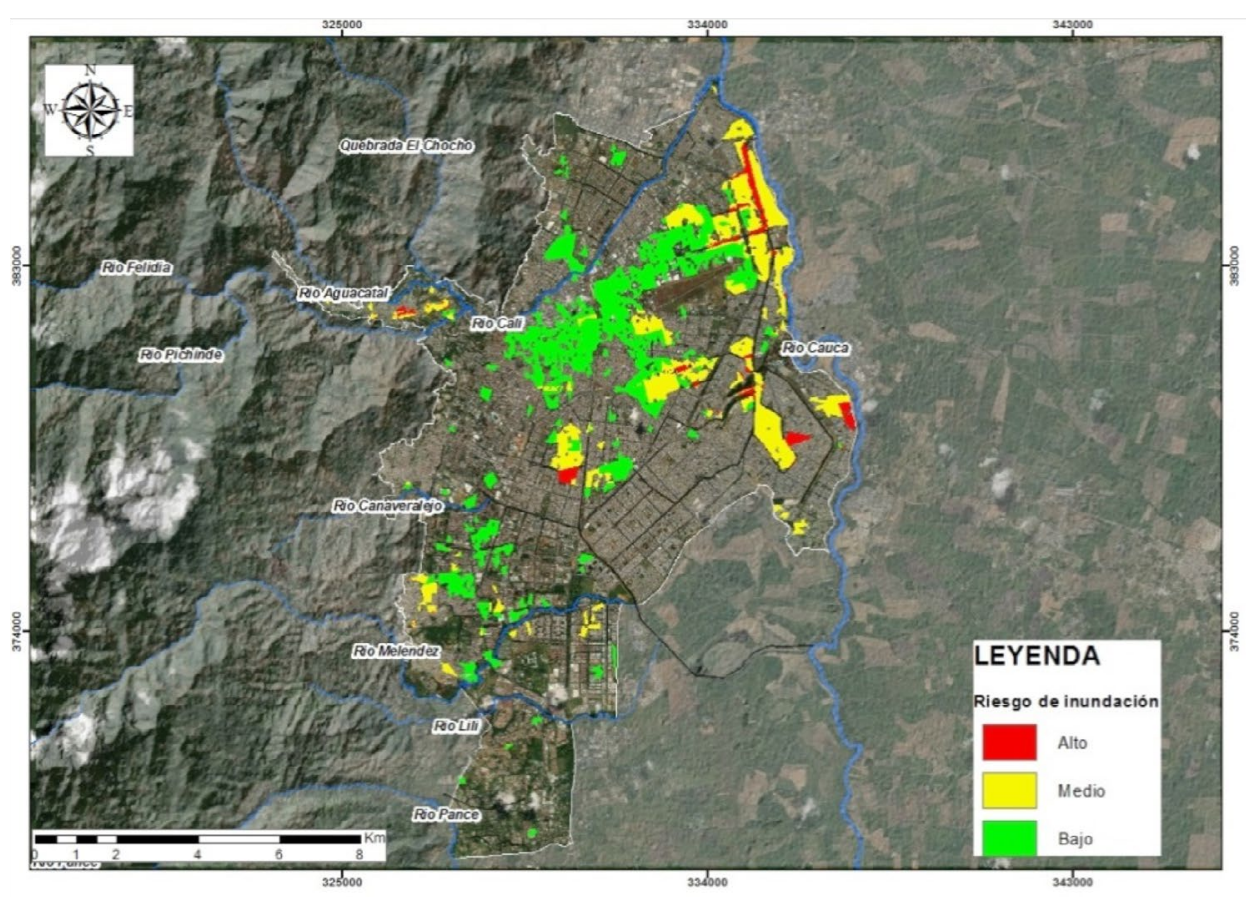

Figura 6. Riesgo por inundación en Cali, Colombia Fuente: Autora, 2021.

La estimación anterior admitió zonificar el riesgo por inundación considerando patrones de amenaza y vulnerabilidad. En la Figura 7, los resultados de cada una de las cartografías. 


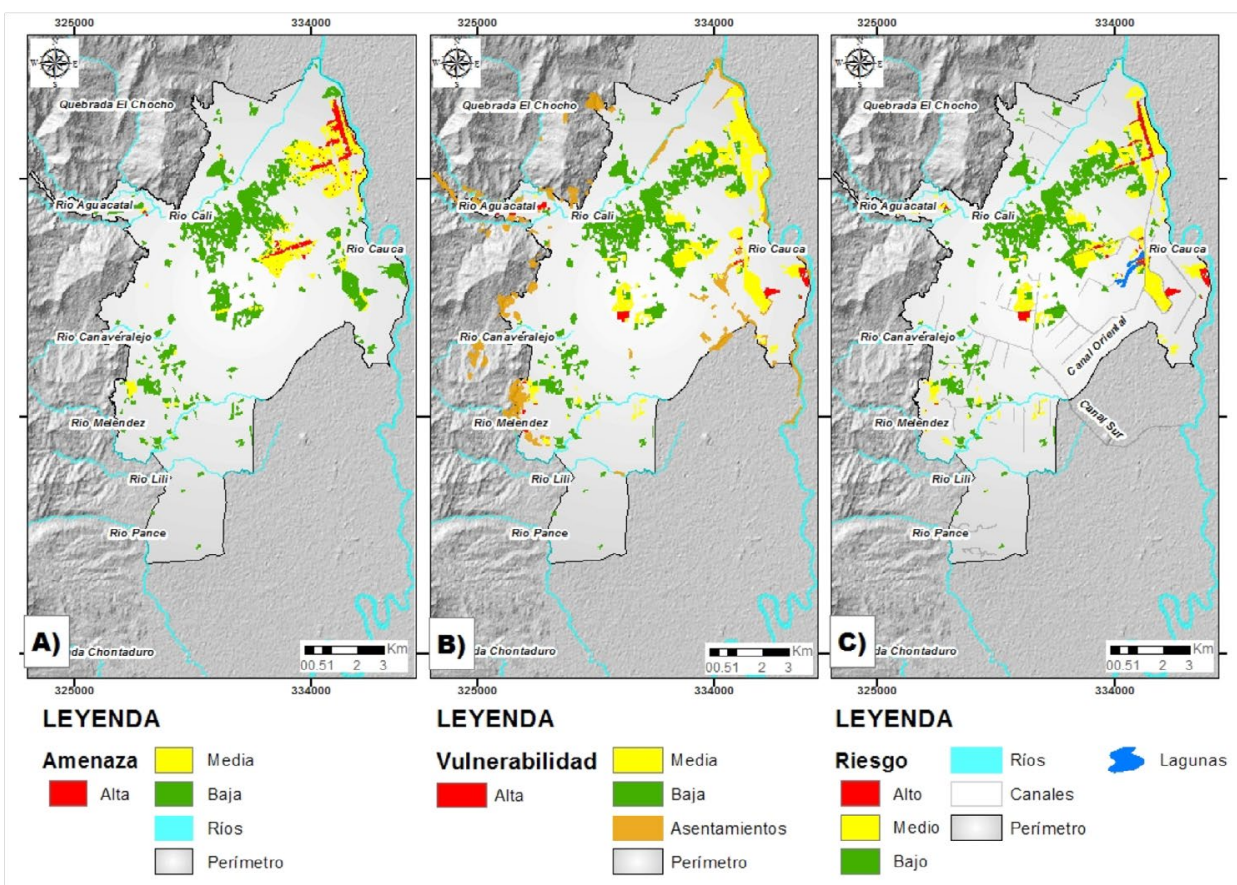

Figura 7. Amenaza, vulnerabilidad y riesgo por inundación

Fuente: Autora, 2021, con base en Sevillano-Rodríguez et al., 2020

La estimación anterior admitió zonificar el riesgo por inundación considerando patrones de amenaza y vulnerabilidad. El siguiente aspecto en la aplicación MESR, fue la valoración de los elementos expuestos. En la Figura 8 se localizó el riesgo alto. El resultado indica la afectación a 34 barrios con diferencia en la extensión e intensidad del fenómeno. De acuerdo a esta información se contabilizaron 37.202 personas que pueden afectarse.

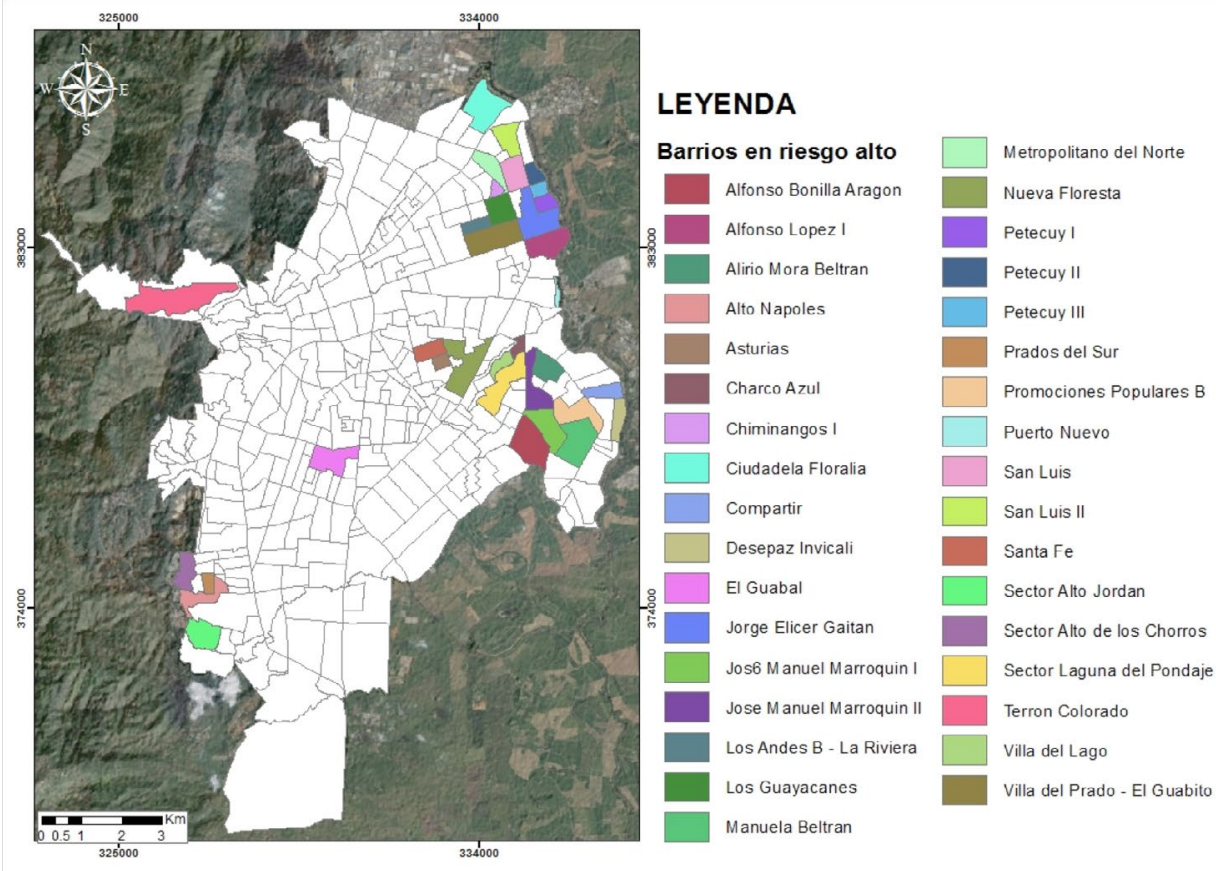

Figura 8. Barrios expuestos a riesgo alto de inundación Fuente: Autora, 2021.

Al mismo tiempo, la revisión de elementos expuestos condujo a determinar la infraestructura esencial para el normal funcionamiento urbano que puede alterarse en riesgo alto y medio. En la Figura 9, los servicios y asentamientos subnormales que podrían sufrir daños. El conteo de elementos expuestos arrojó los asentamientos e infraestructura en situación de riesgo alto y medio: 28 asentamientos (incluidos varios en proceso de desalojo), 2 plantas de agua potable (Empresa 
Emcali: Río Cauca y Puerto Mallarino), 84 vías primarias y secundarias (avenida Ciudad de Cali, autopista Oriental, avenida Nueva granada entre otras), dos hospitales (Joaquín Paz Borrero continuo a quebrada y Mario Correa Rengifo cerda de canal Oriental), 5 torres de comunicación (operadores móviles Claro y Tigo), 5 estaciones de bombeo (entre estas: Paso del comercio, Cañaveralejo, Floralia), 167 instituciones educativas y 74 tuberías de gas (redes principales). Por su parte, la Figura 10 muestra viviendas ubicadas en la margen del río Cauca y tributarios.

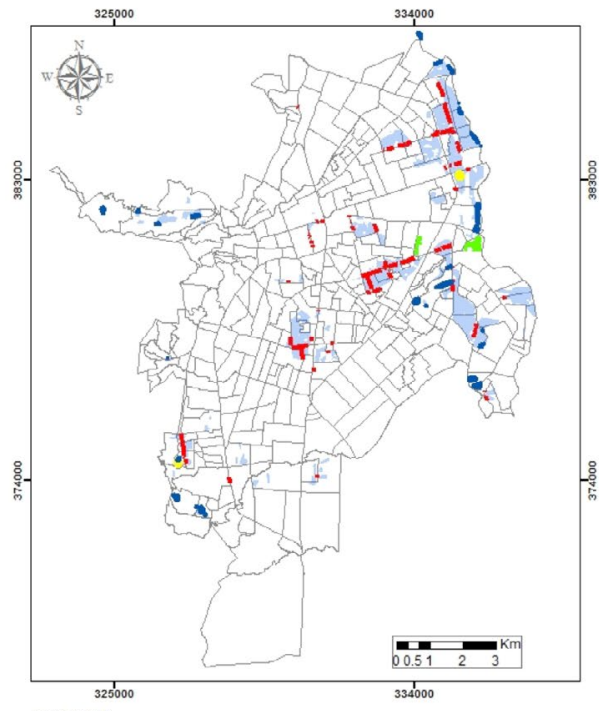

LEYENDA

Asentamientos informales (28)

4 Plantas de agua potable en riesgo alto y medio (2)

- Vias primarias y secundarias en riesgo alto y medio (84)

Hospitales en riego alto y medio (2)

Riesgo alto y medio



Figura 9. Elementos expuestos en riesgo medio y alto Fuente: Autora, 2021.



Figura 10. Viviendas ubicadas en el margen de ríos Fuente: El País (2017).

En la Figura 11, la fotografía de una inundación registrada el 17 de mayo de 2017 a la 
izquierda parte del Oriente Cali; a la derecha Juanchito en el municipio de Candelaria.

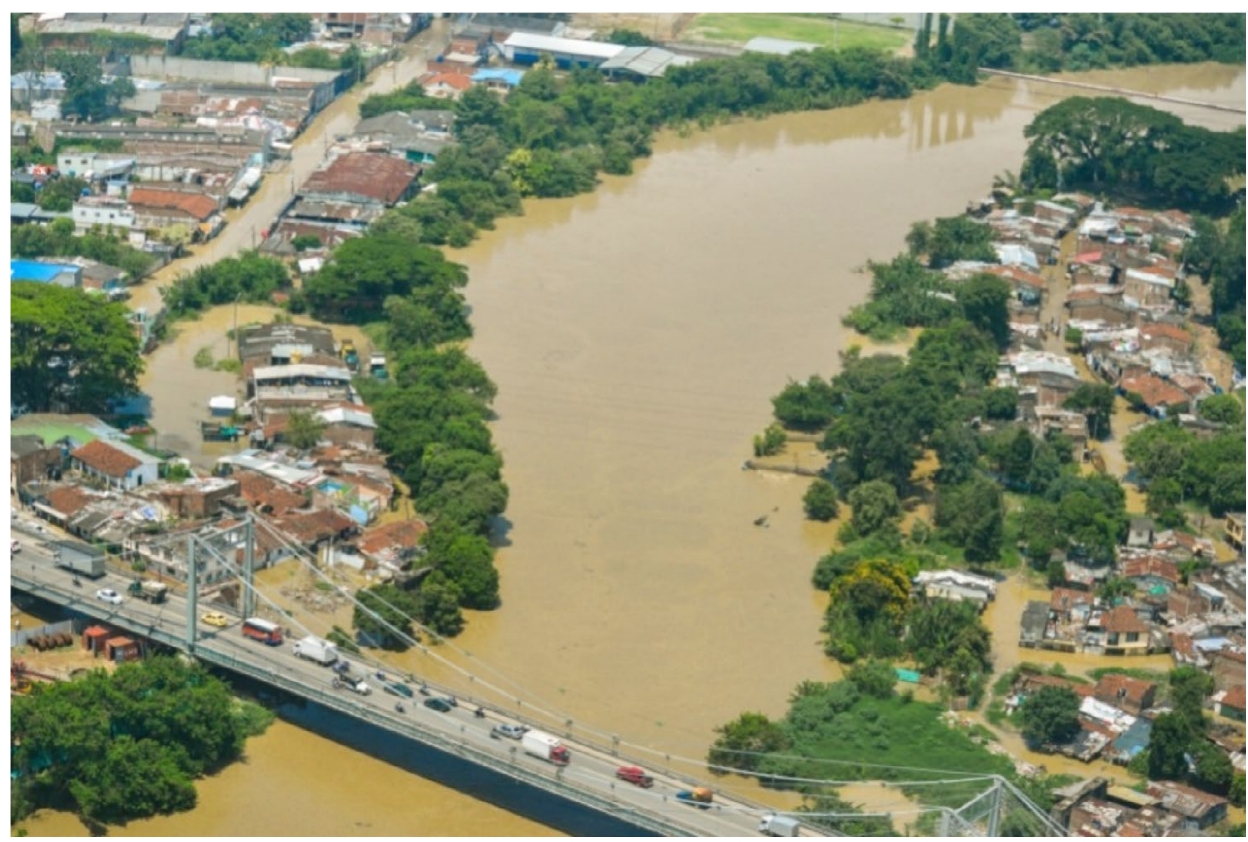

Figura 11. Inundación en Cali y en Candelaria Fuente: El País (2017).

\section{DISCUSIÓN}

El análisis de riesgo de desastres por inundaciones desde la construcción social conduce a valorar condiciones de peligro y vulnerabilidad (Lavell, 1996). De manera que, los desastres son detonados de una situación social, económica y política existente previamente (García-Acosta, 1993). Así, los peligros naturales son los iniciadores del desastre, pero las causas de estos suelen ser múltiples y se basan principalmente en las características socioeconómicas y ambientales de la región impactada (García-Acosta, 1993).

Por consiguiente, estas tipologías determinan la concentración espacial de personas e infraestructura urbana que favorecen un conjunto de relaciones complejas que generan más factores de riesgo (Lavell, 1996). En consecuencia, estas investigaciones cobran importancia y han sido abordadas en ciudades, debido a las tendencias de urbanización y a las condiciones de pobreza que existen primordialmente en países en vía de desarrollo (Park, 2000). Cabe precisar que, estudios anteriores de manera concisa analizaron el riesgo de desastres integrando la amenaza y vulnerabilidad (Wilchex-Chaux, 1993; Blaikie et al., 1996; Cardona, 2001; Cutter et al., 2003).

Los modelos Vulnerabilidad Global, Modelo Presión Liberación, Vulnerabilidad del Lugar y Riesgo Holístico (Wilchex-Chaux, 1993; Blaikie et al., 1996; Cardona, 2001; Cutter et al., 2003), han sido seguidos por diferentes autores con la aplicación de técnicas distintas y variables de análisis que van desde la condición de amenaza o vulnerabilidad. Vale la pena precisar algunos autores que han implementado los métodos considerando las dos condiciones que integran el riesgo de desastre. Utilizando el marco de referencia de Vulnerabilidad Global: Ferrari (2012), consideró la localización del área inundable y factores socioeconómicos, ideológicos y jurídicos para estudiar las inundaciones en la ciudad de Trelew, Argentina; Fernández (2017), utilizó el nivel de amenaza sísmica e indicadores socioeconómicos, educativos y técnicos en la construcción de un índice de vulnerabilidad global para el estado de Sucre, Venezuela. En cuanto al Modelo Presión Liberación: Ley-García \& Calderón-Aragón (2008), revisaron fuentes hemerográficas para identificar elementos históricos de la amenaza y vulnerabilidad en la producción social del riesgo en el periodo 1903-1933 para la ciudad de Mexicali; Guerrero-Amuy (2019), recurrió a la exposición de amenaza volcánica y a factores sociales, físicos, políticos, institucionales y económicos, para determinar la vulnerabilidad en la parroquia de Angochagua, Ibarra, Ecuador. El modelo Peligros del Lugar ha sido replicado en distintas partes del mundo, utilizando indicadores sociodemográficos y análisis de componentes principales que han sido adaptados al conjunto de datos de cada 
localidad. En estados Unidos, Myers et al. (2008), evaluaron la vulnerabilidad social y la migración tras los huracanes Katrina y Rita; en China, Chen et al. (2013), valoraron la vulnerabilidad social a los peligros naturales en el Región del Delta del Río Yangtze; en Noruega, Holand \& Lujala (2013) adaptaron el método SOVI en el análisis de las municipalidades noruegas y realizaron una encuesta para reconocer la percepción frente al riesgo; en España, Pérez-Morales et al. (2016) formularon una propuesta para evaluar la vulnerabilidad social en poblaciones afectadas por el peligro de inundación.

A partir del modelo Holístico, Sierra-González \& Gómez (2008) plantearon una metodología para evaluar el impacto socioeconómico de los desastres en Colombia utilizando datos de sismos, población y pobreza; Torres-Acosta \& Díaz-Parra (2012) evaluaron el riesgo sísmico de la Ciudad de Bogotá; Vera-Rodríguez \& Albarracín-Calderón (2017), propusieron un modelo para el análisis de vulnerabilidad ante amenazas de inundación, remoción en masa y flujo torrenciales en cuencas hidrográficas para la quebrada Cay, Ibagué, Colombia. Habría que decir que, otros autores han desarrollo estrategias metodológicas distintas a las ya mencionadas para ser aplicadas a estudios de caso. Así por ejemplo, Camarasa-Belmonte et al. (2008) propusieron cartografía de riesgo de inundación en llanos del Barranc del Carraixet y de la Rambla de Poyo en España, durante el procedimiento evaluaron la amenaza a partir de datos hidrogeomorfológicos y la vulnerabilidad mediante la combinación de valor económico de suelo, exposición humana y la ocupación del espacio en función de la franja horaria. Por otro lado, Meyer et al. (2008) utilizaron criterios económicos, sociales y ambientales para el río Mulde en Sajonia, Alemania; Shever et al. (2011) integraron dimensiones económicas, sociales y ecológicas para estudiar el riesgo por inundación en Alemania. En el mismo sentido, Dang et al. (2011) aplicaron parámetros económicos sociales y ambientales en la valoración del riesgo por inundación en el área del río Day en Vietnam; MendozaMejía et al. (2014) ocuparon factores físico-naturales y de exposición humana para examinar la vulnerabilidad biofísica en Toluca, México; Siddayao et al. (2014) incorporaron la densidad de población en la evaluación de riesgos de inundación en municipio de Enrile en la provincia de Cagayan, norte de Filipinas.

Por otro lado, Moguel-Flores et al. (2016) propusieron variables de uso de suelo, tipo de vivienda y densidad poblacional para evaluar el riesgo por inundaciones urbanas en Xalapa, México; Shivaprasad-Sharma et al. (2018) clasificaron el riesgo de inundación usando capas de inundación históricas y datos de vulnerabilidad física, social, económica y medio ambiental en la cuenca del río Kopili, Assam, India; Shale-Ogato et al. (2020) se apoyaron en análisis multicriterio y datos de densidad de población para determinar el peligro de inundación y riesgo en Oromia, Etiopía. Conviene subrayar que los trabajos citados arriba tienen en cuenta variables físicas y socioeconómicas, estas pueden ser muy diversas en términos cuantitativos y cualitativos situación que confirma la diversidad interpretativa de los autores (Rufat et al., 2015; Fekete, 2019). Aunque, la construcción de índices no ha sido perfecta, debemos reconocer que aportan elementos de discusión teórica y metodológica. A ello se ha sumado la utilización de sistemas de información geográfica (SIG) para la elaboración de cartografía del riesgo como herramienta para la planificación territorial (Olcina-Cantos \& Díez, 2017).

De ahí que, otro de los métodos ampliamente usado sea la Evaluación Multicriterio (EMC) (Meyer et al., 2008; Shever et al., 2011; Dang et al., 2011; Mendoza-Mejía \& Orozco-Hernández., 2014; Moguel-Flores et al., 2016; Siddayao et al., 2014; Shivaprasad-Sharma et al., 2018; ShaleOgato et al., 2020). Según Rygel et al. (2006), además de la diferencia entre la utilización de variables el principal problema que presenta la aplicación (EMC) es la falta de justificación en la asignación de pesos a indicadores de diferente origen (peligro, vulnerabilidad). En definitiva, la construcción social de riesgo (amenaza-vulnerabilidad) ha sido valorada mediante métodos cuantitativos y cualitativos que han aportado separadamente a la edificación de un enfoque integral con debilidades y fortalezas. De acuerdo a Mendoza-Peñuela \& Sánchez-Calderón (2002), los principales problemas de los estudios de desastres implementados por investigadores sociales es que carecen de un cuerpo teórico y metodológico como sustento. En relación con los estudios de caso Rufat et al. (2015), identificaron dos grupos. Los primeros referidos a investigaciones posteriores al desastre basados en la recopilación de datos empíricos con información detallada en lugares específicos que en sus interpretaciones tienden a generalizar arrojando resultados poco confiables. 
Los segundos, de procesamiento geoespacial con el mapeo y análisis de indicadores cuantitativo que carecen de un contexto y validación. En ambos grupos, la selección de variables, la escala de análisis, la ponderación y la agregación no suelen tener argumentos. En realidad, algunas de las ausencias identificadas en estudios previos fueron recopiladas minuciosamente y contribuyeron al Método de Evaluación Sintetizada para riesgo de Desastre (MESR). Así las cosas, el aporte de este trabajo reside en la aplicación de un método propio en el estudio de riesgo por inundación en Santiago de Cali, Colombia. La implementación del modelo favorece la elaboración de cartografía de riesgo que ayudan en los procesos de planeación y ordenamiento territorial. En este trabajo se tuvo en cuenta la fase de identificación en el proceso de riesgo y se aplicó el Método de Evaluación Sintetizada para riesgo de Desastre (MESR), para evaluar el riesgo por inundación en Santiago de Cali. En el desarrollo del modelo se consideró el peligro de inundación y la vulnerabilidad zonificados anticipadamente. Posteriormente, se aplicó una matriz de combinación (amenaza-vulnerabilidad) que permitió verificar de manera cartográfica el riesgo sintetizado.

En definitiva, se pudieron señalar los elementos expuestos en caso de presentarse una inundación en la ciudad con la probabilidad de un periodo de retorno de 1.1 anual. En consecuencia, se localizaron espacialmente los lugares que se encuentran en riesgo y se clasificaron en tres categorías: alto $118 \mathrm{Ha} \mathrm{(6 \% ),} \mathrm{medio} 749 \mathrm{Ha}(38 \%)$ y bajo $1115 \mathrm{Ha}(56 \%)$. Los puntos de mayor riesgo por inundación se ubicaron al oriente junto al canal Oriental, el río Cauca, las lagunas Charco Azul y el Pondaje; al occidente cerca de los ríos Cali y Aguacatal. Los elementos expuestos en riesgo alto indican la afectación a 34 barrios y 37. 202 personas. Mientras que la integración de riesgo medio y alto podría causar daños a 2 plantas de agua potable, 84 vías, 2 hospitales, 5 torres de comunicación, 5 estaciones de bombeo, 167 instituciones educativas y 74 tuberías de gas. Todos los hallazgos encontrados confirmaron la construcción social del riesgo por inundación, dado que el crecimiento urbano se ha configurado a partir de los ríos, humedales, quebradas, canales y diques que hacen parte de la ciudad. Es decir, muchos habitantes urbanos a causa de situaciones precarias se asentaron sobre las riveras de fuentes hídricas. Además, la construcción de diques y canales favoreció la ocupación del sector oriental donde sus primeros colectivos se valieron de rellenos artesanales para ocupar terrenos que antes conformaban una gran ciénaga.

De igual modo, ciertos asentamientos informales optaron por localizarse encima de los diques, siendo las principales barreras estructurales que favorecen a Cali de inundaciones; cabe anotar que la obstrucción de canales por falta de mantenimiento o por acción humana podría llegar a causar un gran desastre por el desbordamiento del río Cauca. A esto se suma, la incapacidad de canales que empora con las intensas precipitaciones y el vertimiento directo de escombros, residuos sólidos y líquidos; demostrando deficiencias en las relaciones que se presentan entre el hombre y la dinámica urbana. Sin lugar a dudas, esta problemática requiere una mirada institucional que apunte a gestionar y reducir la situación de riesgo de Cali. En este artículo se abordó el enfoque de construcción social (amenaza-vulnerabilidad) aplicando el Método de Evaluación Sintetizada para Riesgo de Desastres con Enfoque de Ordenamiento Territorial (MESR). El desarrollo del modelo facilitó la identificación de riesgo asociado a inundación para la ciudad de Santiago de Cali, Colombia. Debemos añadir que la intención posterior de este tipo de estudios deberá ser el análisis de sus componentes, a partir de estrategias de ordenamiento territorial que propicien la implementación de políticas públicas, por ende, la reducción de los impactos asociados a estos fenómenos.

\section{CONCLUSIONES}

El estudio se centró en aplicar el Método de Evaluación Sintetizada para Riesgo de Desastres con Enfoque de Ordenamiento Territorial (MESR) en la evaluación de vulnerabilidad y riesgo por inundación en la ciudad de Santiago de Cali, Colombia. En este trabajo se siguió el enfoque de construcción social de riesgo asociado a la desigualdad en zonas urbanas. A partir de esta concepción, se integraron mediante una matriz la zonificación de amenaza y de vulnerabilidad sintetizada (indicadores históricos, socioeconómicos y territoriales) y se determinaron los elementos expuestos. De ahí que, los mayores índices de fragilidad tienden a distribuirse espacialmente al Oriente y Occidente de la ciudad. Las mismas zonas, son las que más desastres asociados a movimiento en masa e inundaciones han presentado. 
El riesgo de desastres (Combinación de amenaza y vulnerabilidad) se clasifico en tres categorías: Alto $118 \mathrm{Ha} \mathrm{(6 \% ),} \mathrm{medio} 749 \mathrm{Ha} \mathrm{(38 \% )} \mathrm{y} \mathrm{bajo} 1115 \mathrm{Ha}(56 \%)$. Los sitios de mayor afectación (Riesgo alto) se encuentran al Oriente junto a los canales Interceptor Sur y Oriental, el río Cauca, las lagunas Charco Azul y el Pondaje; al Occidente cerca de los ríos Cali y Aguacatal. Los elementos expuestos indicaron la afectación a 34 barrios, 37.202 personas (Riesgo alto), 28 asentamientos informales, 2 plantas de agua potable, 84 vías y dos hospitales entre otras infraestructuras (Riesgo alto y medio).

La importancia de estos hallazgos reside en la utilidad que tienen para la gestión del riesgo de desastres, estudios que integren factores de amenaza y vulnerabilidad a inundaciones para Cali, donde no se encontraron investigaciones previas que hayan contemplado en una misma cartografía las dos condiciones del riesgo y que además se basen en modelo estructurado bajo distintas clases de vulnerabilidad (histórica, social, económica, territorial). La valoración de vulnerabilidad y riesgo mediante el Método de Evaluación Sintetizada para Riesgo de Desastres con Enfoque de Ordenamiento Territorial (MESR), demostró ser apropiado para este tipo de estudios.

\section{REFERENCIAS}

Acosta-Medina, A.D. (2013). Colombia: Escenario de desigualdades. Revista de la Facultad de Ciencias Económicas y Administrativas, 14(1), 9-35.

Adger, W.N. (2006). Vulnerability. Global Environmental Change, 16(3), 268-281. https://doi. org/10.1016/j.gloenvcha.2006.02.006

Alcaldía Municipal de Santiago de Cali. (2018). Informe anual homicidios. Disponible en: http://www. cali.gov.co/documentos/1201/informes-anual-homicidios/

Alcaldía Municipal de Santiago de Cali. (2014). Plan de Ordenamiento Territorial del municipio de Santiago de Cali. Alcaldía Municipal de Santiago de Cali.

Alcaldía Municipal de Santiago de Cali-DAGMA. (2010). Balance hídrico de las aguas subterráneas: Municipio de Santiago de Cali. Santiago de Cali: DAGMA.

Alcaldía Municipal de Santiago de Cali-IDESC. (2014). IDESC. Recuperado el 1 de enero de 2019, de IDESC: http://idesc.cali.gov.co/geovisor.php

Aneas de Castro, S. (200o). Riesgos y Peligros: Una visión desde la geografía. Scripta Nova, 6o, 55-78.

APFM, OMM \& Asociación Mundial para el Agua. (2009). Gestión Integrada de Crecidas: Documento conceptual. Ginebra: Programa Asociado de Gestión de Crecidas. Recuperado de: http://www.apfm. info/pdf/concept_paper_s.pdf

Blaikie, P., Cannon, T., David, I. \& Wisner, B. (1996). Vulnerabilidad: El entorno social, político y económico de los desastres. LA RED.

Calderón-Ramírez, D. \& Frey, K. (2017). El ordenamiento territorial para la gestión del riesgo de desastres en Colombia. Territorios, 36, 239-264. http://dx.doi.org/10.12804/revistas.urosario.edu.co/ territorios/a.4795

Camarasa-Belmonte, A.M., López-García, M.J. \& García, J.S. (20o8). Cartografía de vulnerabilidad frente a inundaciones en llanos mediterráneos. Caso de estudio del Barranc de Carraixet y Rampla de Poyo. Serie Geográfica, 83(14), 75-91. http://hdl.handle.net/10550/39755

Cardona, O.D. (1993). Evaluación de la amenaza la vulnerabilidad y el riesgo. En A. Mascrey (Ed.), Los desastres no son naturales (pp.1-140). LA RED.

CEPAL. (2002). Políticas públicas para la reducción de la vulnerabilidad frente a los desastres naturales y socionaturales. Santiago de Chile: CEPAL. Recuperado de: https://www.cepal.org/es/ publicaciones/5749-politicas-publicas-la-reduccion-la-vulnerabilidad-frente-desastres-naturales

Chen, W., Cutter, S. L., Emrich, C. T. \& Shi, P. (2013). Measuring Social Vulnerability to Natural Hazards in the Yangtze River Delta Region, China. Int. J. Disaster Risk Sc, 4(4), 169-181. https://doi. org/10.1007/s13753-013-0018-6

Consejo de Europa. (2o de Mayo de 1983). Carta Europa de Ordenación del territorio. Torremolinos, España: Recuperado de: http://cope.sanmartindelosandes.gov.ar/wp-content/uploads/2012/og/ Carta Europea OT.pdf

Cutter, S.L., Boruff, B.J. \& Shirley, W.L. (2003). Social Vulnerability to Environmental Hazards. Social Science Quarterly, 84, 242-261. https://doi.org/10.1111/1540-6237.8402002

Dang, N. M., Babel, M. \& Huynh. (2011). Evaluation of food risk parameters in the Day River Flood Diversion Area, Red River Delta, Vietnam. Natural Hazards, 56(1), 169-194. https://doi.org/10.1007/ 
S11069-010-9558-X

DESINVENTAR. (3 de Mayo de 2018). DESINVENTAR. Recuperado el 3 de Marzo de 2019, de DESINVENTAR: Recuperado de: https://online.desinventar.org/desinventar/\#COL-1250694506colombia inventario historico de desastres

Fekete, A. (2019). Social Vulnerability (Re-) Assessment in Context to Natural Hazards: Review of the Usefulness of the Spatial Indicator Approach and Investigations of Validation Demands. International Journal of Disaster Risk Science, 10, 220-232. https://doi.org/10.1007/s13753-019-0213-1

Fernández, G. (2017). Propuesta metodológica: construcción de un índice de vulnerabilidad global ante la amenaza sísmica, en espacios regionales, con el uso de técnicas estadísticas multivariables. Terra NUEVA ETAPA, 33(54), 127-163.

Fernández, M.A. \& Rodríguez, L. (1996). ¿Cuál es el problema? Introducción a la temática. En M. A. Fernández (Ed.), Ciudades en riesgos: Degradación ambiental, riesgos urbanos y desastres (pp.1-179). LA RED.

Ferradas-Mannucci, P. \& Torres-Guevara, J. (2012). Gestión de riesgos y cambio climático: Relación, tendencias y retos. En F.B. (Coordinador), Perspectivas de investigación y acción frente al cambio climático en Latinoamérica. Número especial de Desastres y sociedad en el marco del XX aniversario de LA RED (pp.1-283). LA RED.

Ferrari, M.P. (2012). Análisis de vulnerabilidad y percepción social. Cuadernos de Geografía: Revista Colombiana de Geografía, 21(2), 99-116.

García-Acosta, V. (1993). Enfoques teóricos para el estudio histórico de los desastres naturales. En A. Mascrey (Ed.), Los desastres no son naturales (pp.1-140). LA RED.

García-Acosta, V. (1997). Historia y desastres en América Latina. Volumen 2. LA RED.

García-Acosta, V. (2005). El riesgo como construcción social y la construcción social del riesgo. Desacatos, 19, 11-24.

Guerrero-Amuy, D.A. (2019). Determinación de la vulnerabilidad volcánica en la parroquia AngochaguaIbarra, Ecuador (Tesis de licenciatura). Ibarra: Universidad Técnica del Norte.

Herzer, H.M. (2011). Construcción del riesgo, desastre y gestión ambiental urbana: Perspectivas en debate. Revista Virtual Redesma. Red de Desarrollo Sostenible y Medio Ambiente, 5(2), 52-6o.

Hewitt, K. (1983). The Idea of Calamity in a Technological Age. En Hewitt, K. (Ed.), Interpretations of Calamity (pp.3-32). A. \&. Unwin.

Hewitt, K. (1996). Daños ocultos y riesgos encubiertos: Haciendo visible el espacio social de los desastres. En E. Mansilla (Ed.), Desastres: Modelo para armar (pp.23-45). LA RED.

Holand, S. \& Lujala, P. (2013). Replicating and Adapting an Index of Social Vulnerability to a New Context: A Comparison Study for Norway. The Professional Geographer, 65(2), 312-328. https://doi.or g/10.1080/00330124.2012.681509

Jiménez, N. (2005). Elementos históricos y urbanos en la generación de desastres por inundaciones y deslizamientos en Cali 1950 - 2000 (Tesis de Licenciatura). Santiago de Cali: Universidad del Valle.

Lampis, A. (2013). Vulnerabilidad y adaptación al cambio climático: debates acerca del concepto de vulnerabilidad y su medición. Cuadernos de geografía, 22(2), 17-33.

Lampis, A. \& Rubiano, L. (2012). ¡Y siguen culpando a la lluvia!. Vulnerabilidad ambiental y social en el sector Altos de la Estancia, Bogotá, Colombia. En F.B. (coordinador), Perspectivas de investigación y acción frente al cambio climático en Latinoamérica. Número especial de Desastres y Sociedad en el marco del XX aniversario de LA RED (pp.1-282). LA RED.

Lavell, A. \& Franco, E. (1996). Estado, sociedad y gestión de los desastres en América Latina. En busca del paradigma perdido. LA RED.

Lavell, A. (1996). Degradación ambiental, riesgos y desastre urbano. Problemas y conceptos: Hacia la definición de una agenda de investigación. En Fernández, M.A. (Ed.), Ciudades en riesgos: Degradación ambiental, riesgos urbanos y desastres (pp.1-179). LA RED.

Ley, J. G. \& Calderón, G. A. (2008). De la vulnerabilidad a la producción del riesgo en las tres primeras décadas de la ciudad de Mexicali, 1903-1933. Región y sociedad, 20(41), 145-173.

Linayo, A. (2012). Cambio climático: La excusa perfecta. En Briones, F. (Ed.), Perspectivas de investigación y acción frente al cambio climático en Latinoamérica. Número espacial de Desastres y 
Sociedad en el marco del XX aniversario de LA RED (pp.1-283). LA RED.

López-Rodríguez, L. (2017). Procedimiento para la simulación hidráulica a partir de los softwareArcGIS y HEC-RAS para eventos extremos máximos (Trabajo de Diploma). Santa Clara: Universidad Central Marta Abreu de Las Villas.

Mansilla, E. (1996). Notas para una reinterpretación de los desastres. En Mansilla, E. (Ed.), Desastres un modelo para armar: Colección de piezas de un rompecabezas social (pp.1-223). LA RED.

Mansilla, E. (2000). La ciudad el nuevo escenario del riesgo. LA RED.

Mansilla, E. (200o). Riesgo y ciudad. LA RED.

Martínez-Rubiano, M. (2011). Perspectiva territorial de la gestión de riesgos de desastres en Colombia. Perspectiva Geográfica, 1(15), 277-298.

Martínez-Rubiano, M.T. (2015). La construcción del conocimiento científico del riesgo de desastres. Epistemología, teorías y metodologías de los estudios desde una perspectiva geográfica (Tesis de Doctorado). Bogotá: Universidad Pedagógica y Tecnológica de Colombia.

Masgrau, L.R. (2004). Los mapas de riesgo de inundaciones: Representación de la vulnerabilidad y aportación de la vulnerabilidad y aportación de las innovaciones tecnológicas. Anál.Geogr, 43, 153-171.

Maskrey, A. (1993). Vulnerabilidad y mitigación de desastres. En Maskrey, A. (Ed.), Los riesgos no son naturales (pp.1-140). LA RED.

Massiris, A. (1999). Ordenamiento territorial. Experiencias internacionales y desarrollos conceptuales y legales realizados en Colombia. Perspectiva Geográfica, 4, 7-70.

Massiris, A. (2002). Ordenación del Territorio en América Latina. Scripta Nova, 6, $105^{-132 .}$

Mendoza-Mejía, J.B. \& Orozco-Hernández, M.E. (2014). Análisis de la vulnerabilidad biofísica a los riesgos por inundación en la zona metropolitana de Toluca, México. Luna Azul, 38, 86-104.

Mendoza-Peñuela, C., \& Sánchez-Calderón, F.V. (2002). Riesgos ambientales: Apuntes para una nueva perspectiva de la construcción social del espacio. Cuadernos de geografía, 11(2), 77-113.

Mendoza-Ramírez, J., \& Aristizábal, E. (2017). Metodología para la zonificación de la susceptibilidad por movimiento en masa en proyectos lineales. Estudio de caso en el acueducto del municipio de Fredonia, Antioquia. Ingeniería y ciencia, 23(26), 163-206.

Metzger, P. (1996). Medio ambiente urbano y riesgos: Elementos de reflexión. En Fernández, M.A. (Ed.), Ciudades en riesgos: Degradación ambiental, riesgos urbanos y desastres (pp.1-179). Lima: LA RED.

Meyer, V., Scheuer, S. \& Haase, D. (2008). A multicriteria approach for flood risk mapping exemplified at the Mulde river, Germany. Natural Hazards, 48, 17-39.

Moguel-Flores, A., Tejeda-Martínez, A. \& García-Pacheco, V. (2016). Propuesta para evaluación de riesgos por inundaciones urbanas: El caso de Xalapa (México). Recuperado de: http://aeclim.org/wpcontent/uploads/2016/02/0096_PU-SA-VIII-2012-AG_MOGUEL.pdf

Muñoz-Robles, C.A., Treviño-Garza, E.J., Verástegui-Chávez, J., Jiménez-Pérez, J. \& Aguirre-Calderón, O.A. (2005). Desarrollo de un modelo espacial para la evaluación del peligro de incendios forestales en la Sierra Madre Oriental de México. Geografía física, 56, 101-117.

Myers, C. A., Slack, T. \& Singelmann, J. (2008). Social Vulnerability and Migration in the Wake of Disaster: The Case of Hurricanes Katrina and Rita. Population and Environment, 29(6), 271-291. https://doi.org/10.1007/s11111-008-0072-y

Narváez, L., Lavell, A. \& Pérez, G. (2009). La Gestión del Riesgo de Desastres. Un enfoque basado en procesos. Lima, Perú: Comunidad Andina. Recuperado de: http://www.comunidadandina.org/ predecan/doc/libros/PROCESOS ok.pdf

NASA. (1o de Marzo de 2018). NASA-EARTHDATA. Obtenido de Recuperado de: https://www.asf. alaska.edu/

Navarro, O., Chaves-Castaño, L., Noreña-Betancur, M.I. \& Piñeres-Sus, J. D. (2016). Percepción del riesgo y estrategias de afrontamiento en población expuesta y no expuesta al riesgo de inundación. Revista Interamericana de Psicología, 50(3), 331-346.

Olcina-Cantos, J. \& Díez-Herrero, A. (2017). Cartografía de inundaciones en España. Estudios Geográficos, 78(282), 283-315.

Park, R. (200o). La ciudad el nuevo escenario del riesgo. En Mansilla, E. (Ed.), Ciudad y riesgo (pp. 
1-181). LA RED.

Pérez-Morales, A., Navarro-Hervás, F. \& Âlvarez-Rogel, Y. (2016). Propuesta metodológica para la evaluación de la vulnerabilidad social en poblaciones afectadas por el peligro de inundación: el caso de Âguilas (Murcia, sureste ibérico). Documents d'Anàlisi Geográfica, 62(1), 133-159.

Pizarro, R. (2001). La vulnerabilidad social y sus desafíos: Una mirada desde América Latina. Santiago de Chile: CEPAL. Recuperado de: https://repositorio.cepal.org/bitstream/handle/11362/4762/ $\underline{\text { So102116 es.pdf }}$

Poinsot, Y. (1992). Algunas consideraciones sobre métodos y conceptos para el ordenamiento territorial. Bogotá, D.C: IGAC. Documento de discusión inédito.

Quarantelli, E. (1996). Desastres y Catástrofes: Condiciones y consecuencias para el desarrollo social. En Mansilla, E. (Ed.), Desastres un modelo para armar: Colección de piezas de un rompecabezas socia (pp.1-223). LA RED.

República de Colombia-DANE. (2005). Censo General de población 2005. República de Colombia - DANE.

República de Colombia - Ley 1523. (24 de abril de 2012). Por la cual se adopta la política nacional de gestión del riesgo de desastres y se establece el Sistema Nacional de Gestión del Riesgo de Desastres y se dictan otras disposiciones. Bogotá, Cundinamarca, Colombia.

República de Colombia-DANE. (2018). Censo general de población 2019. En departamento Nacional de Estadística (DANE). Recuperado de: http://microdatos.dane.gov.co/index.php/catalog/ MICRODATOS/about collection/2/3

República de Colombia-Ley 388. (18 de Julio de 1997). Ley 388 de 1997, Ley de Ordenamiento Territorial. Bogotá, D.C, Colombia.

República de Colombia-UNGRD. (2015). Guía de integración del riesgo de desastres en el ordenamiento territorial. Bogotá: UNGRD. Recuperado de: http://portal.gestiondelriesgo.gov.co/Documents/GuiaIntegracion-Gestion-Riesgo-Ordenamiento-Territorioal-Octubre2015.pdf

República de Colombia-UNGRD. (2018). Unidad Nacional para la gestión del riesgo de desastres. Reportes de inundación 2013-2018 por municipios. Recuperado de: http://portal.gestiondelriesgo. gov.co/

Rodríguez, M.E., Bravo, L.C., Cejudo, L.C. \& Salcedo, E. d. (2020). Identificación de zonas de inundación a partir de imágenes (SAR). Cuadernos Geográficos, 59(2), 308-329. http://dx.doi. org/10.30827/cuadgeo.v59i2.9641

Rodríguez-Esteves, J.M. (2007). La conformación de los „desastres naturales“. Construcción social del riesgo y variabilidad climática en Tijuana. Frontera Norte, 19(37), 83-112. http://www.redalyc.org/ pdf/136/13603704.pdf

Rodríguez-Gaviria, E.M. (2016). Diseño metodológico para la evaluación de riesgo por inundación con información escasa (Tesis de Doctorado). Medellín, Colombia: Universidad Nacional de Colombia.

Rubiano-Vargas, D.M. \& Ramírez-Cortés, F. (2009). Incorporando la gestión del riesgo de desastres en la planificación y gestión territorial. Guía técnica para la interpretación y aplicación del análisis de amenazas y riesgos. Lima Perú: Comunidad Andina. Recuperado de: http://www.comunidadandina.org/StaticFiles/Temas/AtencionPrevencionDesastres/ EJET4IncorporandoGestionRiesgoDesastresPlanificacionGestionTerritorial.pdf

Rufat, S., Tate, E., Burton, C.G. \& Maroof, A. S. (2015). Social vulnerability to floods: Review of case studies and implications for measurement. International Journal of Disaster Risk Reduction, 14, $470-486$.

Rygel, L., O'sullivan, D. \& Yarnal, B. (2006). A Method for Constructing a Social Vulnerability Index. Mitigation and Adaptation Strategies for Global Change, 11, 741-764.

Sandoval, V., \& Sarmiento, J.P. (2018). Una mirada desde la gobernanza del riesgo y la resiliencia urbana en América Latina y el Caribe: Los asentamientos informales en la Nueva Agenda Urbana. Revista de Estudios Latinoamericanos sobre Reducción del Riesgo de Desastres REDER, 2(1), 38-52. http:// revistareder.com/handle-0719-8477-2018-011

Saldaña, L.C., Monsalve-Jaramillo, H., Agudelo-Calvo, J.A., Upegui-Botero, F.M., \& JaramilloFernández, J.D. (2005). Metodología para la evaluación del riesgo sísmico de pequeñas y medianas ciudades. Estudio de caso: Zona centro de la ciudad de Armenia-Colombia. Rev. Int. de Desastres Naturales, Accidentes e Infraestructura Civil, 5(1), 1-20. 
Sevillano-Rodríguez, M.E. (2020). Zonificación de la amenaza ante inundaciones a partir de un método de evaluación multicriterio en la ciudad de Santiago de Cali, Colombia. Geofocus, 25, 47-76. http:// doi.org/10.21138/GF.661

Sevillano-Rodríguez, M.E. \& Bravo-Peña, L.C. (2018). Análisis multitemporal de la expansión física en la ciudad de Santiago de Cali, Colombia. DECUMANOS, 3(3), 28-43.

Shale-Ogato, G., Bantider, A., Abebe, K. \& Geneletti, D. (2020). Geographic information system (GIS)Based multicriteria analysis of flooding hazard and risk in Ambo Town and its watershed, West shoa zone, Oromia regional State, Ethiopia. Journal of Hydrology: Regional Studies, 27, 1-18.

Shever, S., Haase, D. \& Meyer, V. (2011). Exploring multicriteria flood vulnerability by integrating economic, social and ecological dimensions of flood risk and coping capacity: From a starting point view towards an end point view of vulnerability. Natural Hazards, 58(2), 731-751.

Shivaprasad-Sharma, S.V., Parth-Sarathi, R., Chakravarthi, V. \& Srinivasa- Rao, G. (2018). Flood risk assessment using multi-criteria analysis: a case study from Kopili River Basin, Assam, India. Geomatics, Natural Hazards and Risk, 9(1), 79-93. https://doi.org/10.1080/19475705.2017.1408705

Siddayao, G.P., Valdez, S.E. \& Fernández, P.L. (2014). Analytic Hierarchy Process (AHP) in Spatial Modeling for Floodplain Risk Assessment. International Journal of Machine Learning and Computing, 4(5), 450-457.

Sierra-González, S. \& Gómez, D. A. (2008). Propuesta de evaluación de riesgos y desastres. Un enfoque integral. Teoría y Praxis Investigativa, 3(1), 51-58.

Torres-Acosta, A. \& Díaz-Parra, F. (2012). Evaluación Holística del Riesgo Sísmico de la Ciudad de Bogotá. Bogotá.

Urteaga, E. (2011). Modelos de Ordenación del Territorio en Europa: Francia, Alemania y Reino Unido. Estudios Geográficos, 22(270), 263-289.

Vargas, J.E. (2002). Políticas públicas para la reducción de vulnerabilidad frente a los desastres socionaturales. Santiago de Chile: CEPAL. Recuperado de: https://repositorio.cepal.org/bitstream/ handle $/ 11362 / 5749 /$ S2002612 es.pdf? sequence $=1 \&$ is Allowed $=\mathrm{y}$

Vera-Rodríguez, J.M. \& Albarracín-Calderón, A.P. (2017). Metodología para el análisis de vulnerabilidad ante amenazas de inundación, remoción en masa y flujo torrenciales en cuencas hidrográficas. Ingeniería Neogranadina, 27(2), 109-136.

Viand, J. \& Briones, F. (2015). Riesgos al sur. Buenos Aires: LA RED.

Watanabe, M. (2015). Gestión de Riesgo de Desastres en ciudades de América Latina. Apuntes de Investigación, 4, 1-15.

Wilchex-Chaux, G. (1993). Vulnerabilidad global. En Mascrey, A. (Ed.), Los Desastres no son Naturales (pp.1-134). LA RED. 